\title{
THE SIZE AND ORIGIN OF METAL-ENRICHED REGIONS IN THE INTERGALACTIC MEDIUM FROM SPECTRA OF BINARY QUASARS
}

\author{
Crystal L. Martin ${ }^{1,7}$, Evan Scannapieco ${ }^{2}$, Sara L. Ellison ${ }^{3}$, Joseph F. Hennawi ${ }^{4,5,8}$, S. G. Djorgovski ${ }^{6}$, and \\ Amanda P. Fournier ${ }^{1}$ \\ ${ }^{1}$ Department of Physics, University of California, Santa Barbara, CA 93106, USA; cmartin@ physics.ucsb.edu \\ ${ }^{2}$ School of Earth \& Space Exploration, Arizona State University, P.O. Box 871404, Tempe, AZ 85287-1404, USA \\ ${ }^{3}$ Department of Physics and Astronomy, University of Victoria, Victoria, British Columbia, V8P 1A1, Canada \\ ${ }^{4}$ Department of Astronomy, 601 Campbell Hall, University of California, Berkeley, CA 94720-3411, USA \\ ${ }^{5}$ Max-Planck-Institut fur Astronomie, Konigstuhl 17, D-69117 Heidelberg, Germany \\ ${ }^{6}$ Division of Physics, Mathematics, and Astronomy, California Institute of Technology, Pasadena, CA 91125, USA \\ Received 2010 June 3; accepted 2010 July 18; published 2010 August 26
}

\begin{abstract}
We present tomography of the circum-galactic metal distribution at redshift 1.7-4.5 derived from echellete spectroscopy of binary quasars. We find C IV systems at similar redshifts in paired sightlines more often than expected for sightline-independent redshifts. As the separation of the sightlines increases from $36 \mathrm{kpc}$ to 907 $\mathrm{kpc}$, the amplitude of this clustering decreases. At the largest separations, the C IV systems cluster similar to the Lyman-break galaxies studied by Adelberger et al. in 2005. The C IV systems are significantly less correlated than these galaxies, however, at separations less than $R_{1} \simeq 0.42 \pm 0.15 h^{-1}$ comoving Mpc. Measured in real space, i.e., transverse to the sightlines, this length scale is significantly smaller than the break scale estimated previously from the line-of-sight correlation function in redshift space by Scannapieco et al. in 2006. Using a simple model, we interpret the new real-space measurement as an indication of the typical physical size of enriched regions. We adopt this size for enriched regions and fit the redshift-space distortion in the line-of-sight correlation function. The fitted velocity kick is consistent with the peculiar velocity of galaxies as determined by the underlying mass distribution and places an upper limit on the average outflow (or inflow) speed of metals. The implied timescale for dispersing metals is larger than the typical stellar ages of Lyman-break galaxies, and we argue that enrichment by galaxies at $z>4.3$ played a greater role in dispersing metals. To further constrain the growth of enriched regions, we discuss empirical constraints on the evolution of the C IV correlation function with cosmic time. This study demonstrates the potential of tomography for measuring the metal enrichment history of the circum-galactic medium.
\end{abstract}

Key words: cosmology: miscellaneous - galaxies: abundances - galaxies: halos - galaxies: high-redshift intergalactic medium - quasars: absorption lines

Online-only material: color figures

\section{INTRODUCTION}

In essentially every variant of big bang nucleosynthesis, the primordial universe is composed mostly of hydrogen and helium, with trace amounts of the light elements $\mathrm{Li}, \mathrm{Be}$, and $\mathrm{B}$. Heavier elements, the metals, are produced later by stellar nucleosynthesis, and their mass traces the quantity of stars formed. Since it seems reasonable to expect star formation to be confined to galaxies, the discovery of highly ionized carbon (and later highly ionized $\mathrm{Si}$ and $\mathrm{O}$ ) in the tenuous intergalactic hydrogen gas between galaxies came as a considerable surprise (e.g., Meyer \& York 1987; Lu 1991; Songaila \& Cowie 1996). Since these early observations, it has become well established that some enrichment persists to low $\operatorname{Ly} \alpha$ column densities, suggesting at least some filamentary intergalactic structures have been enriched (Ellison et al. 2000; Schaye et al. 2003). These metals are thought to be ejected from galaxies or protogalaxies by powerful galactic winds. These winds circulate metals through a circum-galactic medium (CGM). The formation and evolution of this CGM have not been directly measured.

The history of circum-galactic metal enrichment directly impacts the metallicity of the gas accreted by all galaxies,

\footnotetext{
7 Packard Fellow.

8 NSF Astronomy and Astrophysics Postdoctoral Fellow.
}

the properties of the first generation of stars, and the galactic mass-metallicity relation. Suggestions for when this enrichment took place span a broad range from before the formation of most galaxies by explosions of an early generation of very massive stars (Madau et al. 2001; Qian et al. 2002), to during or just after reionization at $z \approx 5-10$ by low-mass galaxies (Gnedin \& Ostriker 1997; Ferrara et al. 2000; Scannapieco et al. 2002), or even significantly later, redshifts 2-4 (Adelberger et al. 2005b), when the cosmic star formation rate peaked. Observations that constrain the period of metal dispersal are therefore of great interest.

Previously, the evolution of the carbon mass density has provided the primary empirical constraint on intergalactic enrichment. The carbon mass density inferred from the detections of intervening C IV $\lambda \lambda 1548.204,1550.781$, however, depends sensitively on the gas density and the ambient radiation field. In cosmological models, the fraction of carbon in the third ionization state reaches its maximum value near $z \approx 5$ (Oppenheimer et al. 2009). Beyond $z \gtrsim 5$, both the decreasing ionization fraction and the decreasing $\mathrm{C}$ mass density contribute to the decline in the density of C IV systems (Becker et al. 2009; Ryan-Weber et al. 2009). At $z<5$, where the density of C IV systems remains roughly constant (Songaila 2001), substantial intergalactic medium (IGM) enrichment may be masked by the decline in the ionization fraction $n\left(\mathrm{C}^{+3}\right) / n(\mathrm{C})$ with cosmic time (Cooksey et al. 2009; D'Odorico et al. 2010). 
Compared to mass density measurements, measuring the size of enriched regions would be less sensitive to the evolution of the gas density and ambient radiation field. Size measurements require only metal markers, like $\mathrm{C}$ IV detections, in multiple sightlines. They can directly constrain the period of metal dispersal because larger enriched regions at a given cosmic epoch require earlier enrichment (Scannapieco 2005). Size measurements for C IV systems are complicated by both the clustering of galaxies and the degeneracy between Doppler shifts and line-ofsight distance. By comparing previous strategies for measuring the size of enriched regions and introducing a new method in this paper, we aim to establish the viability of size measurements and demonstrate their utility for constraining the history of circum-galactic metal enrichment.

The physical distance winds travel is thought to be relatively independent of galaxy mass and redshift (see, for example, Figure 9 of Oppenheimer \& Davé 2008). The distance at which a wind stalls, and material begins to fall back, defines the size of the metal bubbles surrounding individual galaxies. By circulating metals, galactic winds smooth out the heavy elements over the bubble. Multiple sightlines passing through the same bubble will therefore present metal-line systems that are correlated. The amplitude of this correlation will be roughly constant over all separations smaller than the typical size of the bubble. If bubbles blown by different galaxies overlap, then pairs of sightlines as wide as the scale of the ensemble of coalesced bubbles will present a similar degree of correlation. The typical size of an enriched region may therefore reflect either individual bubbles or groupings of bubbles.

The size of the typical enriched region can be directly inferred from measurements of the spatial clustering of metalline systems. On scales larger than the enriched regions, metalline systems are expected to cluster like their sources. The correlation amplitude for galaxies depends on their halo mass and redshift, i.e., galaxy populations with the same bias cluster the same way. Equivalently, the large-scale clustering of C IV systems determines the bias of the galaxy population dominating enrichment (Scannapieco 2005). The clustering strength of galaxies rises toward smaller scales and steepens on sub-halo scales (Hildebrandt et al. 2009). ${ }^{9}$ At separations smaller than the enriched regions, however, the clustering amplitude of metalline systems will be roughly constant. The scale at which the $\mathrm{C}$ IV correlation function flattens is therefore directly related to the typical size of the enriched regions.

Previously, the clustering of C IV systems could only be measured in redshift space along the line of sight. At large velocity separations, Scannapieco et al. (2006a) showed that the metalline systems cluster like Lyman-break galaxies. At redshift 2.9, the clustering strength of Lyman-break galaxies decreases with increasing separation as $\xi(r) \simeq\left(r / 4.0 h^{-1} \text { comoving Mpc }\right)^{-1.6}$ (Adelberger et al. 2005a). In contrast to this steep rise, at separations less than $150 \mathrm{~km} \mathrm{~s}^{-1}$, the $\mathrm{C}$ IV auto-correlation function presents nearly constant amplitude (Rauch et al. 1996; Pichon et al. 2003; Scannapieco et al. 2006a). This flattening characterizes measured correlation functions for $\mathrm{Mg}$ II and $\mathrm{Si}$ IV as well (Petitjean \& Bergeron 1990; Scannapieco et al. 2006a). To smooth out the metal-line absorption over $150 \mathrm{~km} \mathrm{~s}^{-1}$ in redshift space, enriched regions expanding with the Hubble flow must have physical radii of about $500 \mathrm{kpc} .{ }^{10}$ The more detailed

\footnotetext{
9 This steepening represents the transition from the two-halo term to the one-halo term. It occurs on a somewhat smaller spatial scale than the break in the line-of-sight $\mathrm{C}$ IV auto-correlation function.

${ }^{10}$ At $z \simeq 3$, the rate of cosmic expansion was about $300 \mathrm{~km} \mathrm{~s}^{-1} \mathrm{Mpc}^{-1}$.
}

analysis of Scannapieco et al. (2006a) demonstrated that placing bubbles of metals $\approx 480$ physical $\mathrm{kpc}$ in radius around halos of mass $\approx 10^{12} M_{\odot}$ provided a reasonable fit to the line-of-sight correlation function. This mass scale is similar to the halo mass, $\approx 10^{11.2}-10^{11.8} M_{\odot}$, estimated by Adelberger et al. (2005a) from the clustering of Lyman-break galaxies.

Such a large size for the enriched regions is surprising. First, it is much larger than the galactic impact parameter at which strong $\mathrm{C}$ IV absorption is detected. The $\mathrm{C}$ IV absorption strength declines significantly beyond $b \approx 70-90 \mathrm{kpc}$ (Steidel et al. 2010). Second, it is much larger than the turnaround radii of galactic winds in cosmological simulations at $z \sim 3$ (Aguirre et al. 2001; Theuns et al. 2002; Kawata \& Rauch 2007). In the cosmological simulations of Oppenheimer \& Davé (2008), the bubble radii range from 60 to $100 \mathrm{kpc}$ over a wide redshift range; and the largest bubbles have not formed yet at $z \sim 3$. The analytical model of Furlanetto \& Loeb (2003) predicts that the wind bubbles reach 100 physical kpc at $z \simeq 3$. This discrepancy with the larger size indicted by the line-ofsight clustering of C IV systems motivates our measurement of $\mathrm{C}$ IV clustering between sightlines. In real space, unlike redshift space, distance and velocity information are not mixed. The lineof-sight correlation function may not directly measure physical size if enriched regions have significant velocities with respect to the cosmic expansion. Plausible sources of these velocity kicks include the peculiar velocities of galaxies (Davis \& Peebles 1983), galactic outflows (Martin 2005; Rupke et al. 2005; Weiner et al. 2009; Pettini et al. 2002; Shapley et al. 2003; Steidel et al. 2010), and cold stream accretion (Dekel \& Birnboim 2008).

In this paper, we provide new insight into the distribution of carbon by comparing the redshifts of C IV systems toward binary quasars. Until recently, no suitable sample of binary quasars had been compiled. Lensed sightlines showed highly correlated C IV systems on interstellar scales but do not probe scales larger than a few kpc (e.g., Rauch et al. 1999, 2001, 2002; Ellison et al. 2004). At the other extreme, little correlation was seen in spectra of wide binaries with physical separations $\gtrsim 1.0 \mathrm{Mpc}$ (Coppolani et al. 2006). Spectra of one binary with an intermediate separation of $\approx 50 \mathrm{kpc}$ presented a number of C IV systems common to both spectra (Crotts et al. 1994). Drawing targets from Hennawi (2004) and Hennawi et al. (2006a, 2006b, 2010), we obtained spectra of binary quasars separated by $5^{\prime \prime}-130^{\prime \prime}$ thereby sampling projected separations from 36 to $907 \mathrm{kpc}$, i.e., a range that spans the various suggested scales for the circulation of metals by galactic winds. Our observations differ from those analyzed recently by Tytler et al. (2009) in several important ways. Our sample has a smaller median pair separation (260 kpc versus about $1 \mathrm{Mpc}$ ). Our spectra are more sensitive to weak C IV systems due to their higher resolution. And, we examine the redshift evolution of the clustering properties between $1.7<z<4.5$. (Our median absorption redshift is 3.0 instead of 2.0.) The age of the universe at redshift 4.3 is just 1.4 Myr. Significant enrichment and evolution may occur during the 2.4 Gyr between redshift 4.3 and 1.7. Most importantly perhaps, we introduce the transverse correlation function to describe the clustering of C IV systems. This quantitative description of the metal distribution provides an empirical target (or test) for cosmological models.

The paper is organized as follows. We describe the observations, data quality, and sample of C IV systems in Section 2. In Section 3, we outline several ways to count pairs of C IV systems, compare these pair counts to those expected for uncorrelated absorption systems, and explain how the selection 
function of the survey was modeled. The Appendix derives the expressions for the error in the correlation function measurements. We make the critical comparison between clustering in velocity space and physical space in Section 4 . We discuss the size of metal-enriched regions and the problems with a previously used maximum-likelihood estimator. We place an upper limit on the average outflow speed in Section 4.2. In Section 4.3, we constrain the in situ dispersal of metals at $z \simeq 3$ and argue that the size of the enriched regions requires significant enrichment by winds at an earlier epoch. We propose an evolutionary test in Section 4.4 that may empirically pin down the enrichment history. We summarize the conclusions in Section 5.

Atomic data from Morton (2003) and a cosmology with $\Omega_{m}=0.3, \Omega_{\Lambda}=0.7$, and $H_{0}=70 \mathrm{~km} \mathrm{~s}^{-1} \mathrm{Mpc}^{-1}$ are used unless noted otherwise. At $z=3$, these parameters mean that cosmic expansion produces a velocity shift of $150 \mathrm{~km} \mathrm{~s}^{-1}$ across a physical length of 480 physical kpc, which corresponds to $1.35 h^{-1}$ comoving Mpc.

\section{OBSERVATIONS}

Quasars provide brighter targets for IGM tomography than galaxies do. The separation of lensed quasars is too small, however, to probe circum-galactic scales. Finding binary quasars with appropriate separations for CGM studies required systematic searches near Sloan Digital Sky Survey (SDSS) and $2 \mathrm{dF}$ quasars (Hennawi 2004). The SDSS and $2 \mathrm{dF}$ quasar samples alone contain few binaries at the relevant separations (roughly 5 arcsec up to an arcminute) because the optical fibers of spectrographs cannot be placed this close. At proper transverse separations $l<1 h^{-1}$ Mpc, Hennawi et al. (2006a) identified 221 new quasar pairs with $0.5<z_{\mathrm{QSO}}<3.0$. Another 27 high-redshift binaries, $2.9<z<4.3$, were recently confirmed to have proper transverse separations from $10 \mathrm{kpc}$ to $650 \mathrm{kpc}$ (Hennawi et al. 2010). From these samples, we selected bright binary quasars that had similar redshifts. Our targets are listed in Table 1. They form 29 pairs, including one triplet.

At the W. M. Keck Observatory, we obtained spectra of 55 quasars with the Echellete Spectrograph and Imager (ESI; Sheinis et al. 2002). The echellete format provided continuous spectral coverage from $4000 \AA$ to $10000 \AA$ at $60 \mathrm{~km} \mathrm{~s}^{-1}$ (filled slit) resolution. Exposure times were chosen to reach a signalto-noise ratio $(\mathrm{S} / \mathrm{N})$ of $15-20$ per pixel. This spectral quality provides sensitivity to $C$ IV components with column density $N\left(\mathrm{C}_{\text {IV }}\right) \approx 10^{13} \mathrm{~cm}^{-2}$.

The ESI spectra were reduced using the ESIRedux ${ }^{11}$ data reduction pipeline. Scattered light was fitted and subtracted from the spectroscopic flat fields. One-dimensional spectra were extracted for each order and averaged using an $\mathrm{S} / \mathrm{N}$ weight function that varied smoothly across the order. Division of each spectral order by the fitted quasar continuum left flat, normalized orders that were combined into a one-dimensional spectrum. Figure 1 shows example spectra of two quasars separated by 100 physical kpc.

\subsection{Sample of C IV Systems}

In each quasar spectrum, we identified C IV absorption lines by the doublet spacing. We required the two transitions have consistent velocity structure. In a few cases, this revealed blends between $\mathrm{C}$ IV and unrelated lines. We searched the bandpass between the Ly $\alpha$ forest and the observed wavelength of C IV at

\footnotetext{
11 http://www2.keck.hawaii.edu/inst/esi/ESIRedux/index.html
}

the quasar redshift. Each pair of spectra probes C IV absorption over a similar redshift interval because the quasars were selected to have roughly the same redshift.

For illustration, the 1548 and 1551 transitions are marked by solid and dotted lines, respectively, in the J0117+3153 spectra in Figure 1. For absorption troughs with well separated 1548 and $1551 \AA$ lines, as seen, for example, at $z=$ $1.9987,2.0540,2.4018$, and 2.4961 toward J0117+3153-A, we directly integrated the equivalent width in each line, $W_{\mathrm{obs}}=$ $\int 1-I(\lambda) / I_{c}(\lambda) d \lambda$. We fitted a pair of Gaussian line profiles to measure the absorber redshift.

When the 1548 and $1551 \AA$ lines were blended, we could not directly integrate their equivalent widths. Multiple velocity components were typically required to model these broad absorption troughs. We fitted Gaussian profiles using the SPECFIT program. ${ }^{12}$ We defined C IV systems by linking components separated by less than $50 \mathrm{~km} \mathrm{~s}^{-1}$, the effective spectral resolution (for the atmospheric seeing). We set the system redshift equal to the weighted average of the component redshifts. The system's equivalent width was calculated from the formula above using the fitted model of the absorption trough in place of the data. Linking yielded 316 intervening $\mathrm{C}$ IV systems and 134 associated systems. Associated systems lie within $5000 \mathrm{~km} \mathrm{~s}^{-1}$ of the QSO (in the same sightline) and will not be included in the sample discussed in this paper.

Figure 2 shows the redshift of intervening CIV systems along each sightline. The paired sightlines are ordered by the separation of the binary quasars. The median sightline separation is $260 \mathrm{kpc}$ with a range from $36 \mathrm{kpc}$ to $907 \mathrm{kpc}$. The absorber redshifts range from 1.7 to 4.3 with a median of 3.0. The spectra of $\mathrm{J} 1420+28, \mathrm{~J} 1021+11, \mathrm{~J} 1541+27$, and $\mathrm{J} 1622+07$ provide critical coverage of small spatial scales beyond the median redshift.

\subsection{Distribution Function}

As discussed in detail previously (Ellison et al. 2000; Songaila 2001; Scannapieco et al. 2006a), the number of C IV systems in a quasar spectrum increases rapidly with improved sensitivity to column density. Most of the systems in our sample with rest equivalent width, $W_{r}(1551)$, less than $200 \mathrm{~m} \AA$ are optically thin. Their measured doublet ratio, $W_{\text {obs }}(1548) / W_{\text {obs }}(1551)$, is consistent with 2 . Their C IV column density follows directly from their equivalent width, $N(\mathrm{C}$ IV $) \approx 10^{14.0} \mathrm{~cm}^{-2}\left(W_{r, 1551} / 200 \mathrm{~m} \AA\right)$. This relation provides only a lower limit on the column density of optically thick systems, however. At the modest dispersion of our spectra, optically thick lines need not be black at line center. We identified many optically thick systems by their smaller doublet ratio, $1 \leqslant W_{\text {obs }}(1548) / W_{\text {obs }}(1551)<2$. We therefore describe the distribution of line strengths by their equivalentwidth distribution instead of their column density distribution.

Distribution functions for intervening absorbers are usually normalized by redshift path rather than redshift. A population with constant comoving space density and constant proper size produces an equal number of systems per unit redshift path, $X(z)$, along a sightline. For the cosmology adopted here, we have $d X \equiv(1+z)^{2}\left[\Omega_{\Lambda}+\Omega_{m}(1+z)^{3}\right]^{-0.5} d z$ (Scannapieco et al. 2006a). We used our sample of intervening $C$ IV systems to compute the equivalent width distribution function, $f(W)$, defined as the number of systems found per unit redshift path per unit rest equivalent width as follows.

\footnotetext{
12 A brief description is given by Kriss (1994).
} 
Table 1

ESI Spectroscopy

\begin{tabular}{|c|c|c|c|c|c|c|}
\hline $\begin{array}{l}\text { Paired Quasars } \\
\text { (1) }\end{array}$ & $\begin{array}{c}z \text { QSO } \\
\text { Redshift } \\
(2)\end{array}$ & $\begin{array}{c}r \\
(\mathrm{AB}) \\
(3)\end{array}$ & $\begin{array}{c}\theta \\
(") \\
(4) \\
\end{array}$ & $\mathrm{S} / \mathrm{N}$ & Date Observed & $\begin{array}{l}\tau \\
(\mathrm{s}) \\
(7) \\
\end{array}$ \\
\hline SDSSJ0117+3153B & 2.640 & 19.86 & 11.3 & 29.0 & $2005 \mathrm{Nov}$ & 17280 \\
\hline SDSSJ0117+3153A & 2.625 & 20.52 & $"$ & 18.5 & 2005 Nov & 17280 \\
\hline SDSSJ0225+0048C & 2.700 & 20.40 & $100.3,75.3$ & 12.8 & 2006 Nov, 2008 Jan & 6771 \\
\hline SDSSJ0225+0048A & 2.820 & 20.54 & $100.3,27.4$ & 12.6 & 2005 Nov & 7100 \\
\hline SDSSJ0225+0048B & 2.757 & 20.40 & $75.3,27.4$ & 16.5 & 2008 Jan & 7260 \\
\hline SDSSJ0245-0113A & 2.462 & 19.63 & 4.5 & 20.2 & $2006 \mathrm{Nov}$ & 12400 \\
\hline SDSSJ0245-0113B & 2.459 & 20.51 & $"$ & 16.5 & 2006 Nov & 12400 \\
\hline SDSSJ0818+0719A & 4.618 & 19.66 & 132.9 & 19.8 & 2006 Nov & 1800 \\
\hline SDSSJ0818+0718B & 4.178 & 20.08 & “ & 19.8 & 2008 Jan & 1800 \\
\hline SDSSJ0925+3859A & 3.135 & 19.32 & 57.1 & 22.5 & 2005 Nov & 6105 \\
\hline SDSSJ0925+3859B & 2.823 & 20.11 & $"$ & 20.8 & 2005 Nov & 10800 \\
\hline SDSSJ0956+2643A & 3.083 & 19.28 & 16.5 & 19.0 & 2007 Apr & 2400 \\
\hline SDSSJ0956+2643B & 3.083 & 20.52 & $"$ & 14.8 & 2007 Apr & 14740 \\
\hline SDSSJ0959+1033A & 4.021 & 19.34 & 44.1 & 33.8 & 2005 Nov & 12500 \\
\hline SDSSJ0959+1033B & 4.021 & 20.11 & $“$ & 11.2 & 2006 Nov & 6300 \\
\hline SDSSJ1021+1112A & 3.830 & 20.60 & 7.6 & 45.7 & 2007 Apr & 21600 \\
\hline SDSSJ1021+1112B & 3.830 & 20.71 & $"$ & 24.8 & 2007 Apr & 21600 \\
\hline SDSSJ1026+4614A & 3.421 & 19.73 & 37.1 & 24.0 & 2008 Jan & 3600 \\
\hline SDSSJ1026+4614B & 3.345 & 19.73 & $“$ & 17.0 & 2008 Jan & 3600 \\
\hline SDSSJ1116+4118A & 3.000 & 18.18 & 13.8 & 33.3 & 2006 Mar & 3291 \\
\hline SDSSJ1116+4118B & 3.000 & 19.19 & $"$ & 18.0 & $2006 \mathrm{Mar}$ & 3291 \\
\hline SDSSJ1144+0959A & 3.146 & 18.33 & 23.5 & 33.6 & $2007 \mathrm{Apr}$ & 2400 \\
\hline SDSSJ1144+0959B & 2.974 & 19.87 & & 23.1 & 2007 Apr & 10800 \\
\hline SDSSJ1228+0232A & 3.138 & 20.42 & 47.3 & 11.7 & 2008 Jan & 3600 \\
\hline SDSSJ1228+0232B & 3.095 & 19.83 & “ & 11.3 & 2008 Jun & 2400 \\
\hline SDSSJ1248+1957A & 3.876 & 20.23 & 64.8 & 14.6 & 2008 Mar, 2008 Jun & 4800 \\
\hline SDSSJ1248+1957B & 3.817 & 19.55 & $“$ & 16.5 & 2008 Mar, 2008 Jun & 3000 \\
\hline SDSSJ1307+0422A & 3.024 & 18.05 & 8.2 & 55.3 & 2007 Apr & 3600 \\
\hline SDSSJ1307+0422B & 3.024 & 19.33 & $"$ & 22.3 & $2007 \mathrm{Apr}$ & 3600 \\
\hline SDSSJ1314+2818A & 4.821 & 21.27 & 116.6 & 18.6 & $2008 \mathrm{Jul}$ & 2400 \\
\hline SDSSJ1314+2818B & 4.390 & 20.92 & “ & 7.5 & $2008 \mathrm{Jul}$ & 900 \\
\hline SDSSJ1353+4852A & 3.848 & 20.43 & 37.1 & 10.1 & $2008 \mathrm{Jul}$ & 2400 \\
\hline SDSSJ1353+4852B & 3.848 & 20.91 & “ & 15.2 & $2008 \mathrm{Jul}$ & 3600 \\
\hline SDSSJ1420+2830A & 4.312 & 20.33 & 10.9 & 20.8 & 2008 Jun & 10800 \\
\hline SDSSJ1420+2830B & 4.290 & 20.77 & $“$ & 20.5 & 2008 Jun & 10800 \\
\hline SDSSJ1513-0131A & 3.224 & 19.23 & 48.1 & 22.0 & 2007 Apr & 4200 \\
\hline SDSSJ1513-0131B & 3.191 & 19.91 & $"$ & 18.1 & 2007 Apr & 7200 \\
\hline SDSSJ1627+4606A & 4.110 & 20.66 & 34.1 & 20.4 & 2007 Apr & 10800 \\
\hline SDSSJ1627+4606B & 3.812 & 20.15 & $"$ & 23.1 & 2007 Apr & 9600 \\
\hline SDSSJ1054+0216A & 3.976 & 19.53 & 88.2 & 16.2 & 2007 Apr & 2400 \\
\hline SDSSJ1054+0216B & 3.976 & 18.81 & $"$ & 19.8 & 2007 Apr & 2400 \\
\hline SDSSJ1215-0309A & 4.001 & 20.20 & 47.1 & 13.3 & 2007 Apr & 2400 \\
\hline SDSSJ1215-0309B & 3.672 & 19.81 & $"$ & 15.4 & 2007 Apr & 2400 \\
\hline SDSSJ1404+4005A & 4.027 & 19.62 & 47.3 & 17.6 & 2007 Apr & 2556 \\
\hline SDSSJ1404+4005B & 4.027 & 21.01 & 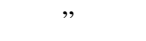 & 13.3 & 2007 Apr & 7200 \\
\hline SDSSJ1541+2702A & 3.621 & 20.62 & 6.4 & 14.1 & 2008 Jan, 2008 Mar & 13782 \\
\hline SDSSJ1541+2702B & 3.626 & 20.71 & “ & 10.4 & 2008 Jan, 2008 Mar & 13782 \\
\hline SDSSJ1542+1733A & 3.261 & 20.06 & 27.4 & 15.2 & 2008 Jun & 3000 \\
\hline SDSSJ1542+1733B & 2.782 & 18.87 & “ & 18.5 & 2008 Jun & 1200 \\
\hline SDSSJ1622+0702A & 3.256 & 20.33 & 5.8 & 14.9 & $2008 \mathrm{Jul}$ & 3000 \\
\hline SDSSJ1622+0702B & 3.260 & 17.19 & “ & 86.5 & $2008 \mathrm{Jul}$ & 3000 \\
\hline SDSSJ1627+2215B & 3.251 & 20.55 & 35.6 & 11.4 & 2008 Jun & 5400 \\
\hline SDSSJ1627+2215A & 3.709 & 19.29 & “ & 12.7 & 2008 Jun & 3000 \\
\hline SDSSJ2157+0015A & 2.560 & 20.55 & 20.8 & 12.7 & $2006 \mathrm{Nov}$ & 4257 \\
\hline SDSSJ2157+0015B & 2.542 & 19.39 & “ & 11.6 & 2006 Nov & 3000 \\
\hline
\end{tabular}

Notes. (1) Quasar name. (2) Quasar redshift. As described in Hennawi et al. (2010), we measure the wavelengths of C IV, C III, and $\mathrm{Si}$ IV and apply the typical shift found between these high-ionization lines and the systemic frame, as traced by the Mg II doublet (Shen et al. 2007). (3) Quasar $r$-band magnitude. (4) Angular separation of pair. Two values indicate the triplet system. (5) Median continuum $\mathrm{S} / \mathrm{N}$ in the bandpass between Ly $\alpha$ and $\mathrm{C}$ IV; the continuum within $5000 \mathrm{~km} \mathrm{~s}^{-1}$ of either line is excluded from the distribution. (6) Date observed. (7) Total exposure time. 


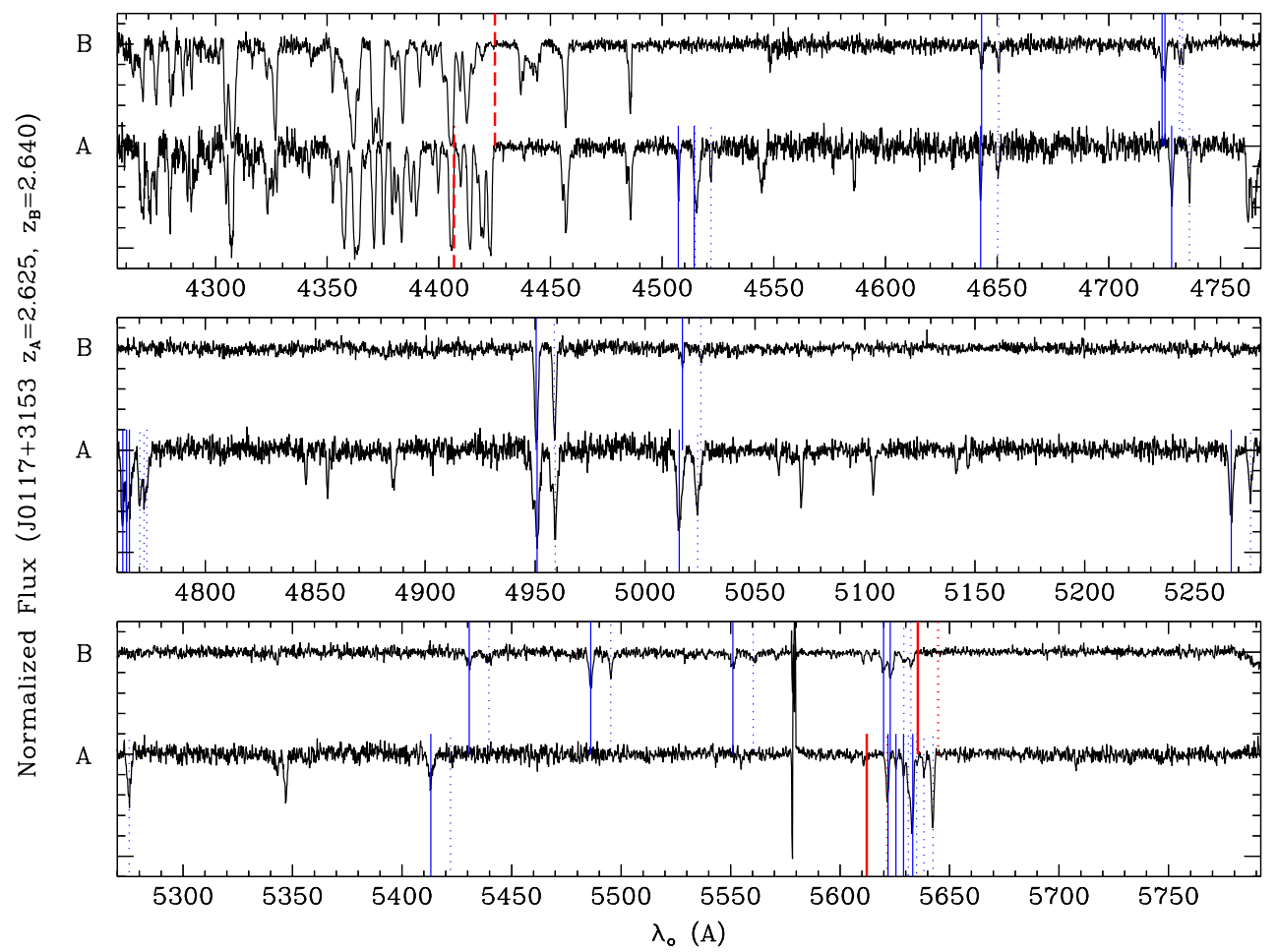

Figure 1. Example of sightlines separated by 100 physical kpc. Thick, vertical lines (red) mark Ly $\alpha$ and $\mathrm{C}$ IV at the quasar redshift. Thin vertical lines (blue) mark each C IV system. We consider all pairs of C IV systems between sightline $A$ and sightline $B$. Provided $\Delta v<c$, their velocity separation is $\Delta v=c\left(1+z_{A B}\right)^{-1}\left|z_{A}-z_{B}\right|$, where $z_{A} B$ is the average redshift of the two systems. A small velocity separation identifies coincident systems. The number of coincident system greatly exceeds the number we would find in the absence of any correlation between the sightlines.

(A color version of this figure is available in the online journal.)

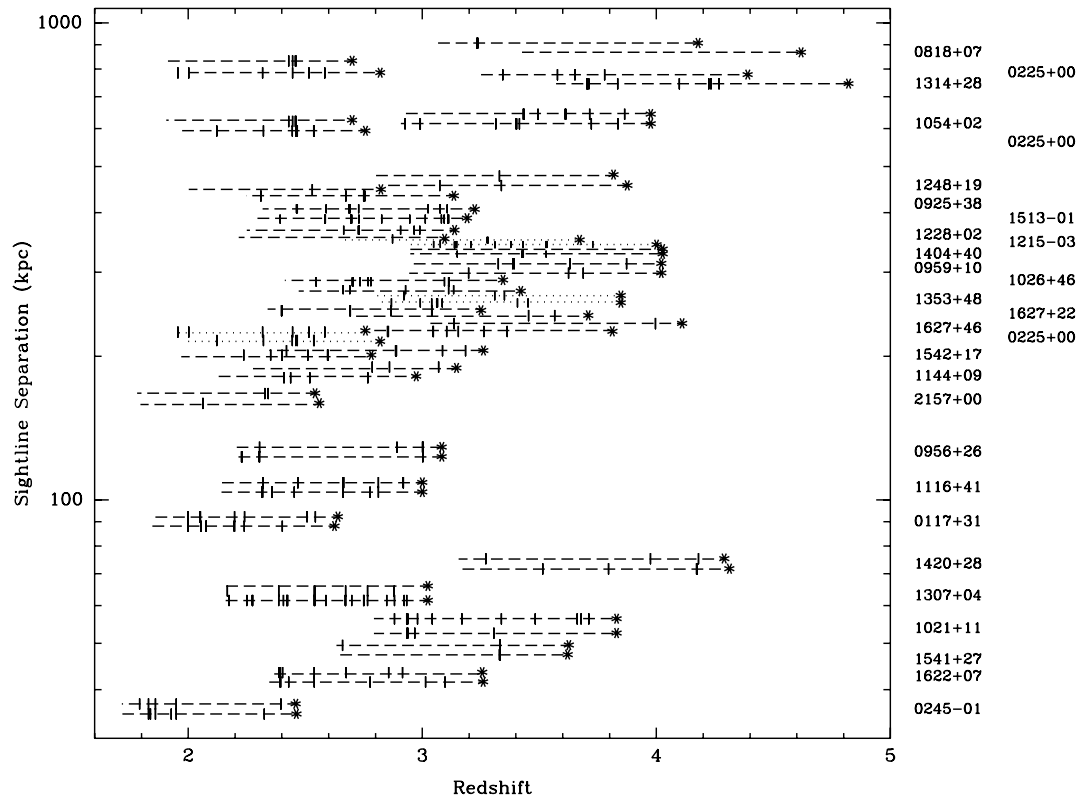

Figure 2. Sightlines as a function of binary quasar separation and redshift probed. Each sightline is shown at the separation of the binary quasar. The quasars are marked by asterisks. A vertical tick marks C IV systems along each sightline. (Small vertical offsets have been applied to prevent overlapping symbols.)

For each C IV system, $i$, in our sample, we calculated the total redshift path over which the system could have been detected, $\Delta X_{i}=\sum_{j=1}^{N_{\text {Qso }}} \Delta X_{i, j}$. Detection at redshift $z$ requires the observed equivalent width of the weaker $\mathrm{C}$ IV transition exceed the $5 \sigma$ measurement uncertainty for a system of velocity width $230 \mathrm{~km} \mathrm{~s}^{-1}$ at the observed wavelength. ${ }^{13}$ This width is

\footnotetext{
13 Spectra containing the wavelength dependence of the continuum $\mathrm{S} / \mathrm{N}$ for each spectrum are available from the lead author
}

typical of a system near the median equivalent width in our sample. For each equivalent-width bin, we calculate the number of systems per unit redshift path,

$$
\left(\frac{d \mathrm{~N}}{d X}\right)_{k}=\sum_{i=1}^{\mathrm{N}_{k}} \frac{1}{\Delta X_{i}}
$$

where $\mathrm{N}_{k}$ is the number of systems in bin $k$. Normalization by the bin width, $\Delta_{k} \log W_{r, 1551} \equiv \log W_{\text {max, } \mathrm{k}}-\log W_{\text {min,k }}$, yields 


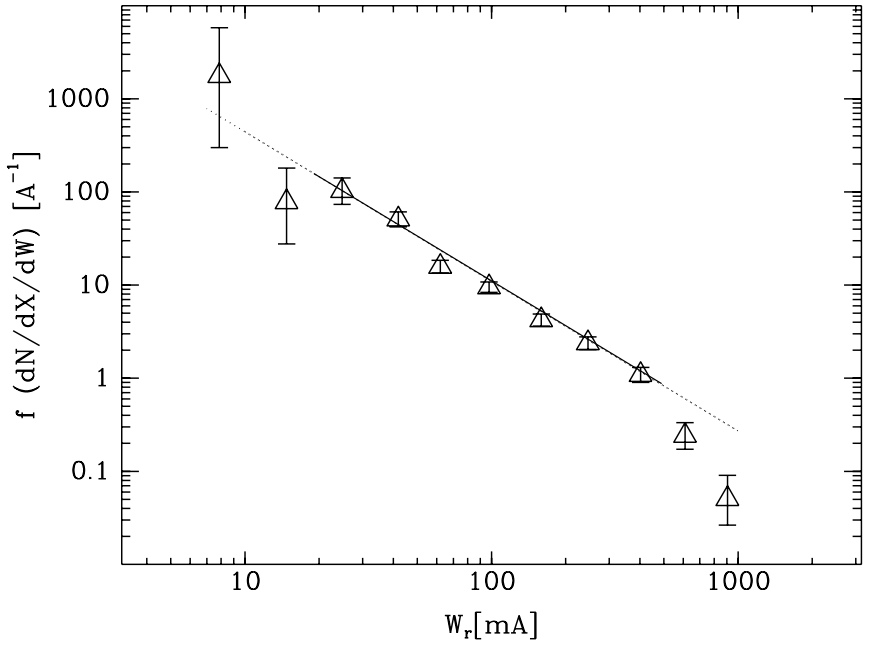

Figure 3. Equivalent-width distribution function for intervening C IV 1551. Components have been grouped into systems using a linking length of $50 \mathrm{~km} \mathrm{~s}^{-1}$. We fit the distribution function with a power law of the form, $\log f\left(\AA^{-1}\right)=b+m \log W(m A)$, including errors in $W$ equal to one-half the bin width, see the text. The uncertainty $\delta f_{k}$ follows from the upper and lower $84.1 \%$ confidence level Poisson limits, corresponding to $1 \sigma$ limit for Gaussian statistics, on the number of objects in bin $k$. We find $b=4.3 \pm 0.4$ and $m=-1.6 \pm 0.2$; or, for $W$ in $\AA, b=-0.6 \pm 0.4$.

the distribution function

$$
f\left(\langle W\rangle_{k}\right)=\Delta_{k}^{-1}\left(\frac{d \mathrm{~N}}{d X}\right)_{k}
$$

at the mean (rest-frame) equivalent width,

$$
\log \langle W\rangle_{k} \equiv \mathrm{N}_{k}^{-1} \sum_{i=1}^{\mathrm{N}_{k}} \log W_{i}
$$

of systems in that bin. Figure 3 shows the resulting distribution function. Over nearly two decades in equivalent width, it is well described by a power law. We attribute the turnover at large equivalent width, $W_{r, 1551} \gtrsim 500 \mathrm{~m} \AA$, to the nonlinear relation between equivalent width and column density. The relatively small redshift path observed at the highest sensitivity causes the large error bars at $W_{r, 1551}<20 \mathrm{~mA}$. The fit to the seven points between 20 and $500 \mathrm{~m} \AA$ is shown in Figure 3. This fit to the distribution function is used in our Monte Carlo simulations in Section 3. At low equivalent width, a direct comparison can be made to the column density distribution functions. We find consistency with Ellison et al. (2000), Songaila (2001), and Scannapieco et al. (2006a).

\section{CORRELATION FUNCTIONS FOR C IV SYSTEMS}

Two approaches are used here to describe the clustering of $\mathrm{C}$ IV systems. The first requires multiple sightlines at relatively small separations. The second, which has been applied previously, estimates separation in redshift space instead of real space. The two methods therefore measure different physical quantities. This section describes the first method in some detail and applies both approaches to the data. Readers interested primarily in the insight gleaned from comparing the two methods may skip directly to Section 4.

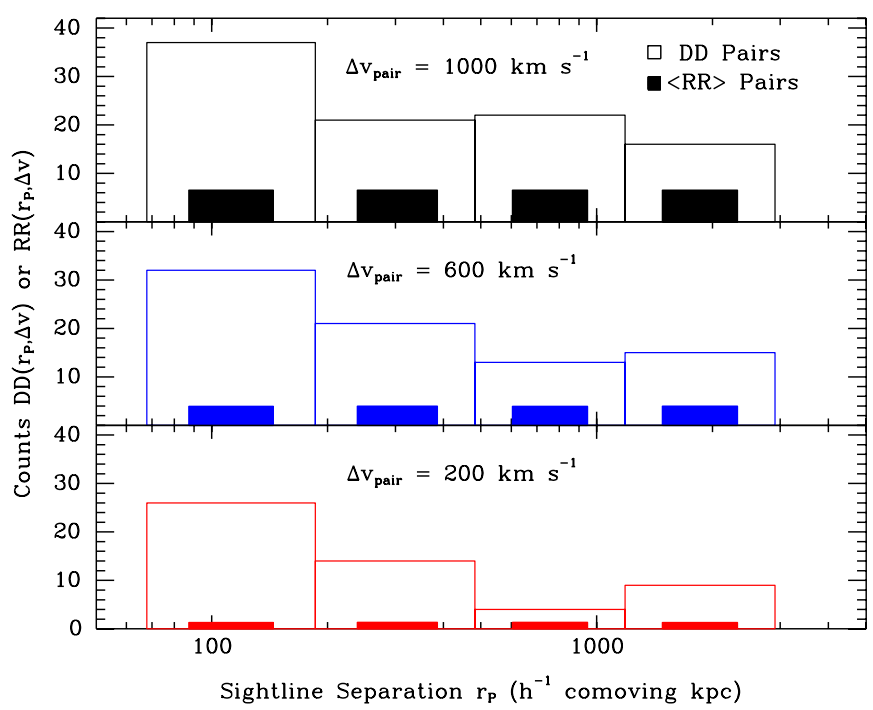

Figure 4. Number of pairs of C IV systems as a function of sightline separation, $r_{P}$. For each CIV system in sightline $A$, we computed the velocity separation between it and every $C_{\text {IV }}$ system in sightline $B$. Velocity separation smaller than a threshold, $\Delta v_{\text {pair }}$, identify coincident systems-pairs of C IV systems at nearly the same redshift in both sightlines. We sum the number of coincident systems over all the binary quasars $l$ in the bin so that $D D\left(r_{P}, \Delta v_{\text {pair }}\right) \equiv \sum_{l} D D^{l}\left(r_{P}, \Delta v_{\text {pair }}\right)$. The data (open histogram) present many more systems at similar redshift than would be expected from uncorrelated sightlines (filled histogram). The bin boundaries were chosen to have a similar number of random-random pairs, where the expectation value, $R R\left(r_{P}, \Delta v_{\text {pair }}\right) \equiv \sum_{l}\left\langle R R^{l}\left(r_{P}, \Delta v_{\text {pair }}\right)\right\rangle$ was obtained from a Monte Carlo simulation. From bottom to top, the three panels show how the number of coincident systems declines as the definition of a pair is relaxed to include larger velocity differences.

(A color version of this figure is available in the online journal.)

\subsection{Cross-correlation of C IV Systems between Sightlines}

\subsubsection{Pair Counts}

The number of absorption-line systems found at similar redshift, but in different sightlines, is related to the correlation function on the scale of the sightlines separation. To find this relationship, data-data pairs can be defined by the apparent velocity difference of the C IV systems in two sightlines. For example, in Figure 1, pairs of C IV systems are detected in sightlines $A$ and $B$ at $\lambda 4642, \lambda 4728, \lambda 4950$, and $\lambda 5015$. Whether or not the system at $\lambda 5412$ in sightline $A$ and the system at $\lambda 5430$ in sightline $B$ count as a pair depends on the velocity threshold used in the definition. In this example, their velocity separation is about $950 \mathrm{~km} \mathrm{~s}^{-1}$, where $\Delta v=$ $c\left(1+z_{A B}\right)^{-1}\left|z_{A}-z_{B}\right|$ and $z_{A} B$ is the average redshift of the two systems. Histograms of pair counts are illustrated in Figure 4 for three values of the threshold velocity, $\Delta v_{\text {pair }}=200,600$, and $1000 \mathrm{~km} \mathrm{~s}^{-1}$. The counts of systems separated by $\Delta v_{A B}<\Delta v_{\text {pair }}$ have been summed over all pairs $l$ of sightlines at similar transverse separation $r_{P}$. For the most inclusive definition, all five pairs detected toward $\mathrm{J} 0117+3153$ contribute to the second bin at $300 h^{-1}$ comoving Mpc. At fixed transverse separation, the number of data-data pairs declines with more restrictive pair definitions, i.e., smaller $\Delta v_{\text {pair. }}$ For any value of $\Delta v_{\text {pair }}$, however, the counts in Figure 4 increase as the separation of the pairs decreases.

We can statistically differentiate physically related pairs of C IV systems from chance coincidences. The number of unrelated pairs, hereafter random-random pairs $R_{A} R_{B}\left(r_{P}, \Delta v_{\text {pair }}\right)$, was modeled using a Monte Carlo simulation. The velocity 


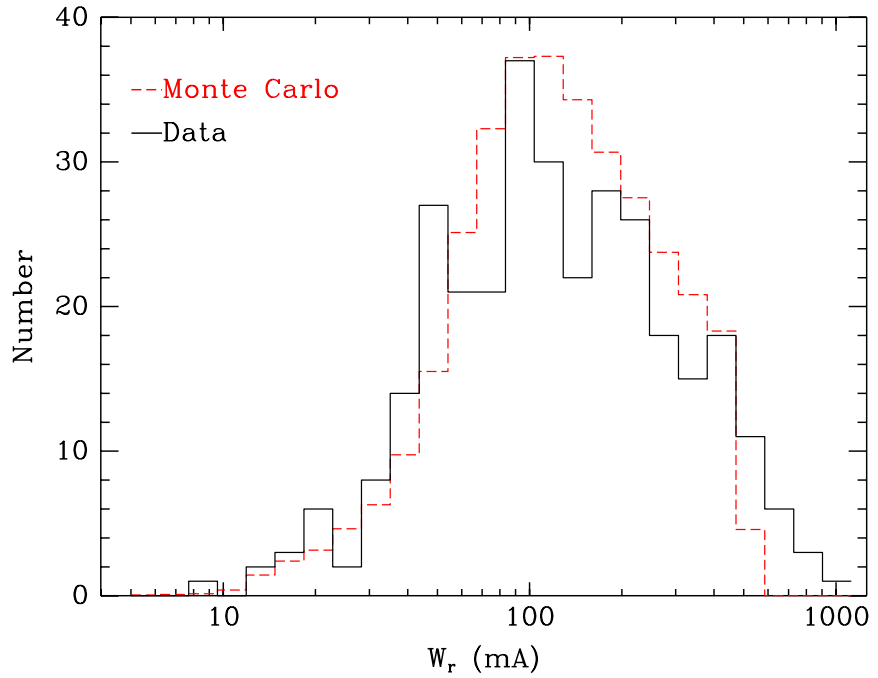

Figure 5. Distribution of accepted C IV $W_{r, 1551}$ equivalent widths. Dashed, red histogram compares the fake absorbers to the data, represented by the solid histogram. The distribution function for fake absorbers was cutoff at $500 \mathrm{~m} \AA$ in the Monte Carlo simulation, and the comparison is void for stronger systems. The fake and real data are consistent within their uncertainties, where the statistical error in the fake data is $3.1 \%$ after 1000 Monte Carlo iterations.

(A color version of this figure is available in the online journal.)

widths of the fake systems were fixed at $230 \mathrm{~km} \mathrm{~s}^{-1}$. Equivalent widths were drawn from the distribution function shown in Figure 3. The redshifts of the fake systems were randomly chosen within the survey range for each quasar sightline. In the Monte Carlo simulations, fake systems were rejected if the observed-frame equivalent width was less than the $5 \sigma$ detection limit. Because the variance is a strong function of observed wavelength in each spectrum, and spectral quality varied by sightline, the redshift distribution of the fake systems reflects the sample biases introduced by telluric features, quasar emission lines, and intervening absorption lines. The equivalent widths of the fake systems are shown in Figure 5. Their distribution appears consistent with the real sample. Averaged over 1000 iterations of the simulation, the average number of fake systems in a given sightline agrees (to within the Poisson error) with the actual number of C IV systems found. When we compare sightlines, these fake systems yield far fewer pairs of absorbers.

In each bin of the histogram shown in Figure 4, the ratio of data-data pairs, $D D\left(r_{P}, \Delta v_{\text {pair }}\right)$ to random-random pairs $R_{A} R_{B}\left(\Delta v_{\text {pair }}\right)$ defines a cross-correlation amplitude, where $\xi_{A B}+$ $1=D_{A} D_{B} / R_{A} R_{B}$. If $D_{A} D_{B}=R_{A} R_{B}$, then all the pairs are random coincidences; and no clustering is detected. The pair counts are implicitly a function of the binning, so we drop the explicit reference to the binning. The variance in the ratio $D D / R R$ is generally larger than the Poisson error in the bin counts. The correction to the Poisson term is significant when $D D>N_{\text {abs }} / 4$ (Mo et al. 1992). In most bins, the number of $D D$ pairs is high compared to the number of absorbers, $N_{\mathrm{abs}}$, in sightlines contributing to the bin. Calculating the uncertainty of the measured correlation therefore required special attention.

Landy \& Szalay (1993) introduced the estimator, $\xi=$ $(D D-2 D R+R R) / R R$, to minimize the variance in a correlation function. For the cross-correlation of sightlines, the mean number of data-random pairs is $\langle 2 D R\rangle \equiv\left\langle D_{A} R_{B}\right\rangle+\left\langle D_{B} R_{A}\right\rangle$, where the average is taken over 1000 fake sightlines with the same selection function as the corresponding quasar spectrum. The correlation strength, $\xi_{A B}\left(r_{P}, \Delta v_{\text {pair }}\right)$, calculated from these

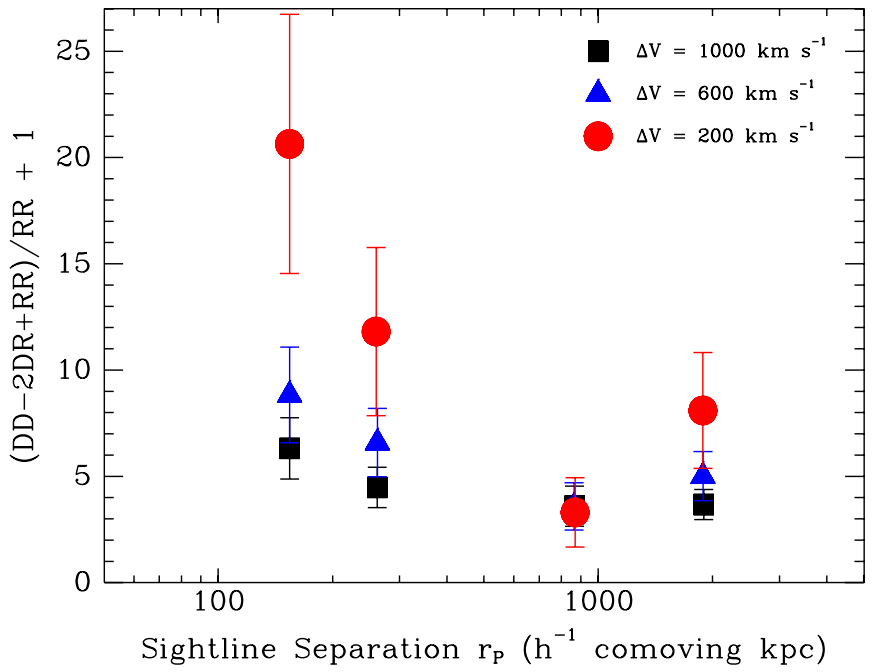

Figure 6. Cross-correlation amplitude of CIV systems vs. the separation of the sightlines. The value of the Landy \& Szalay (1993) estimator is shown at the median separation in each bin. The $1 \sigma$ error bars include a correction to the Poisson term as described in the Appendix. Decreasing the maximum velocity difference defining pairs of $\mathrm{C}$ IV systems, $\Delta v_{\text {pair }}$, increases the correlation amplitude between the sightlines. For each value of $\Delta v_{\text {pair }}$, the correlation amplitude declines as the sightline separation increases.

(A color version of this figure is available in the online journal.)

pair counts is shown in Figure 6. Since $\xi>1$ for these data, the Landy \& Szalay estimator does not reduce the variance to the Poisson level. The Appendix explains how we computed the correction to the Poisson term. These larger error estimates are shown for all correlation function figures.

In Figure 6, the widest sightlines at $r_{P}=2.844 h^{-1}$ comoving $\mathrm{Mpc}$ present highly correlated C IV systems. The systems cluster even more strongly as the sightline separation decreases toward $0.075 h^{-1}$ comoving Mpc. The amplitude of the correlation is independent of the chosen binning in $r_{P}$.

As $\Delta v_{\text {pair }}$ increases, however, the correlation becomes weaker in Figure 6. Stated another way, the probability that C IV systems (in different sightlines) are physically related declines as their redshift separation increases. This sensitivity of clustering strength to $\Delta v_{\text {pair }}$ presents an obstacle for comparing the clustering of C IV systems to other populations.

\subsubsection{Transverse Correlation Function}

One measure of clustering that can be evaluated for our sample, and compared to other populations, is the projection of the redshift-space correlation function. The projection of $\xi^{\prime}(r)$ along the line of sight is of particular interest. In redshift space, the separation of two systems is $r^{2}=r_{P}^{2}+\Delta \Pi^{2}$. The distance in redshift space is calculated from the velocity difference of two systems,

$$
\Delta \Pi \equiv(1+z) \Delta v / H(z),
$$

in comoving units. The integration limits can be chosen to integrate over peculiar velocities, thereby robustly identifying physically related pairs of C IV systems. This projection

$$
w\left(r_{P}, \Pi_{\text {pair }}\right) \equiv \int_{-\Pi_{\text {pair }}}^{+\Pi_{\text {pair }}} \xi^{\prime}\left(r_{P}, \Pi\right) d \Pi
$$

defines the transverse correlation function. It differs from the angular correlation function in that we use the comoving 


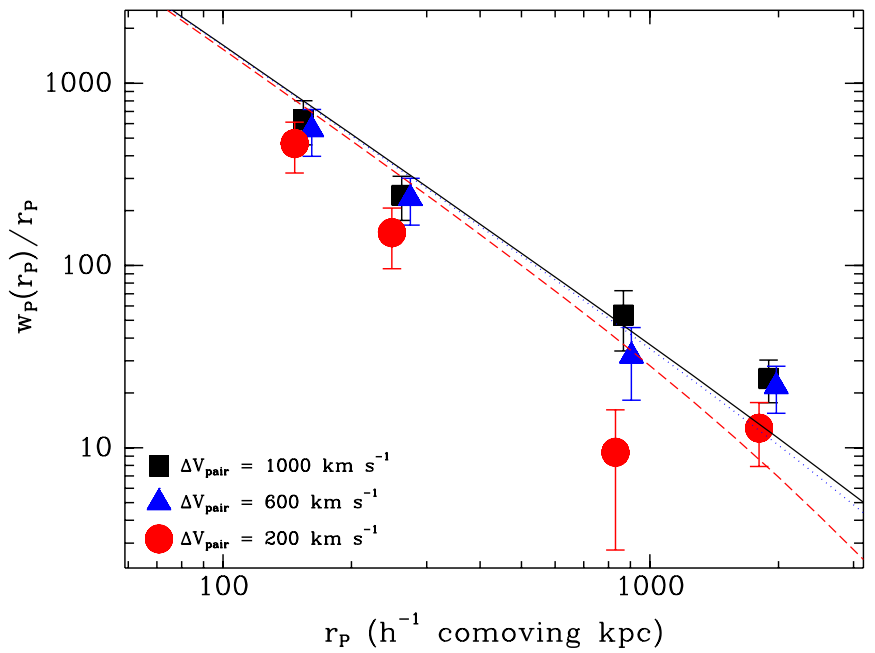

Figure 7. Transverse C IV correlation function normalized by sightline separation vs. median sightline separation. The bins are identical to those in Figures 4 and 6 . The lines show the projection of the galaxy-galaxy correlation function at $z \simeq 2.9$ (Adelberger et al. 2005b), which has been extrapolated below $1 h^{-1}$ comoving Mpc with constant power-law index $\gamma=1.6$ and perturbed by linear infall (Hawkins et al. 2003). Because infall reduces the volume in redshift space, it increases the correlation amplitude slightly. Independent of projection interval, however, the clustering amplitude of C IV systems is very similar to that of Lyman-break galaxies. No parameters are fitted in this figure, so their similarity demonstrates that these galaxies and C IV systems have nearly the same correlation length.

(A color version of this figure is available in the online journal.)

distance transverse to the sightlines $r_{P}(z)$, rather than the angle between the sightlines, to describe separation in the plane of the sky. This approach makes sense because $r_{P}(z)$ is nearly constant over the projection interval of interest.

Figure 7 shows the transverse correlation function. It is the product of $\xi_{A B}\left(r_{P}, \Delta v_{\text {pair }}\right)$ and the (comoving) distance $2 \Delta \Pi_{\text {pair }}$. Since the transverse correlation function $w$ has dimensions of length, we normalizing it by the sightline separation and plot the dimensionless quantity $w / r_{P}$. The C IV correlation amplitude increases as the sightline separation decreases. For the two points at the smallest separations, the amplitudes of $w / r_{P}$ are essentially identical for the three projection intervals illustrated. At larger separations the correlation strength presents a measurable, although still small, dependence on the projection interval.

To illustrate the influence of the projection interval on $w / r_{P}$, consider the projection of Lyman-break galaxies. Their clustering amplitude in redshift-space $\xi^{\prime}(r)$ is higher than $\xi(r)$ in real space because cosmological infall causes structures to appear smaller in redshift space than their true physical size. The reduced volume in redshift space increases the clustering amplitude. To estimate the boost to the galaxy-galaxy correlation function, we apply Equation (15) of Hawkins et al. (2003) which describes linear infall. ${ }^{14}$ The magnitude of the correction depends on the bias. Figure 7 shows the projection for $b=\sigma_{8, g} / \sigma_{8, \mathrm{CDM}}=2.4, r_{0}=4.0 h^{-1}$ comoving Mpc, and $\gamma=1.6$ (Adelberger et al. 2005a). At sightline separations $\gtrsim 1 h^{-1}$, this infall correction noticeably boosts the correlation amplitude for each projection interval $\Delta \Pi$; but it has no detectable effect at smaller separations. The galaxy-galaxy corre-

14 Although linear infall theory is not strictly valid on these scales, Cooray \& Sheth (2002) argue that it is a good approximation. lation function is not fitted to the CIV correlation function in Figure 7. It is therefore remarkable that the amount of C IV clustering agrees roughly with the amplitude of the galaxy-galaxy correlation function.

Previous work revealed a strong cross-correlation amplitude between Lyman-break galaxies and C IV systems on scales $\gtrsim 1 h^{-1}$ comoving Mpc (Adelberger et al. 2005b). We would like to explore the relationship of galaxies and C IV systems on the smaller scales probed by the binary quasars, but this turns out to be difficult with current data. Adelberger et al. (2005a) only measured the galaxy-galaxy correlation function at separations greater than $1 h^{-1}$ comoving Mpc. On sub-halo scales, the galaxy-galaxy correlation function is expected to be steeper than it is in the two-halo regime. By extrapolating the $\gamma=1.6$ power law fitted on large scales, the amplitude of the transverse correlation function for Lyman-break galaxies is a lower limit at small separation in Figure 7. This lower limit for galaxies is higher than the upper limit (one standard deviation) for C IV systems at small scales. In light of the uncertain magnitude of this discrepancy, this result alone is hardly compelling evidence for a difference in the clustering properties of Lyman-break galaxies and C IV systems on small scales.

We observed only a few binary quasars at separations greater than $1 h^{-1}$ comoving Mpc, so our data alone poorly constrain the power-law index of the correlation function on large scales. Taking $\gamma=1.6$ (like Lyman-break galaxies) as a prior, the fitted correlation length $r_{0}=3.65 \pm 0.34 h^{-1}$ comoving Mpc for our sample technically agrees with the value $r_{0}=4.0 \pm 0.6$ fitted by Adelberger et al. (2005a). The lower value, however, reflects a best fit with a lower correlation amplitude overall. This difference is worth noting because our sightlines at separations greater than $1 h^{-1}$ comoving Mpc show a slightly stronger correlation in Figure 7 than do galaxies. There seem to be two options. Our measurement can be fit with a larger correlation length $r_{0}=7.0 \pm 2.0 \mathrm{~h}^{-1}$ comoving Mpc and shallow powerlaw index $\gamma=1.0 \pm 0.2$. Or, we can adopt that prior that C IV systems cluster like Lyman-break galaxies on large scales and find that $\mathrm{C}$ IV do not cluster as strongly as Lyman-break galaxies on scales $\lesssim 1 h^{-1}$ comoving Mpc. To resolve this ambiguity, we re-examine the clustering of $C_{\text {IV }}$ systems along the line of sight before returning to the best fit to the transverse correlation function in Section 4.1.

\subsection{Verification of the Line-of-sight Correlation Function}

For each quasar spectrum (and fake spectrum), we calculated the velocity separations $\Delta v$ of the C IV systems. The separations of data-data, data-random, and random-random pairs were binned. When we sum over all sightlines $l$, the counts yield the correlation strength

$$
\xi_{\mathrm{LOS}}(\Delta v)=\frac{\sum_{l} D_{A} D_{A}-2 \sum_{l} D_{A} R_{A}+\sum_{l} R_{A} R_{A}}{\sum_{l} R_{A} R_{A}}
$$

As discussed previously for $\xi_{A B}$, this estimator has a larger error than the Poisson term when $\xi>1$. The Appendix describes our error estimate. These errors are shown on our correlation function in Figure 8. The clustering amplitude grows steadily as the velocity difference of the pairs decreases from 500 to $100 \mathrm{~km} \mathrm{~s}^{-1}$. Below separations of about $100 \mathrm{~km} \mathrm{~s}^{-1}$, the clustering strength is constant. Our measurement confirms the break described by Scannapieco et al. (2006a). Their spectra, obtained at higher spectral resolution with UVES, demonstrated the near constant amplitude down to the linking length. 


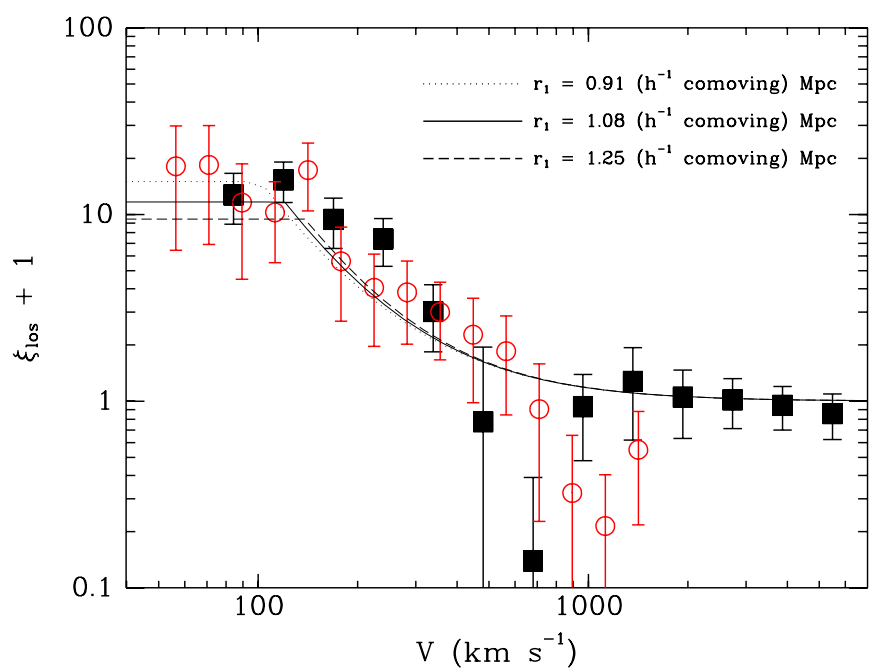

Figure 8. Two-point correlation function of $\mathrm{C}$ IV absorption systems vs. lineof-sight distance. The new ESI data (squares) agree with the earlier VLT data (red circles). The C IV components shown in Figure 10 of Scannapieco et al. (2006a) were grouped into systems using a linking length of $50 \mathrm{~km} \mathrm{~s}^{-1}$. The bins near $500 \mathrm{~km} \mathrm{~s}^{-1}$ have a small number of systems; and we attribute the low pair counts to systematic error introduced by the C IV doublet spacing. Fixing $r_{0}=4.0 h^{-1}$ comoving $\mathrm{Mpc}$ and $\gamma=1.6$ in Equation (7), we fit $r_{1}=1.08 \pm 0.17 h^{-1}$ comoving Mpc to the joint data (solid line); the dashed and dotted lines illustrate the $1 \sigma$ error in $r_{1}$.

(A color version of this figure is available in the online journal.)

We fit the line-of-sight correlation with a simple model. Any model of the auto-correlation function for C IV systems must break at small separations to fit the relatively flat correlation amplitude at small velocity separation. Scannapieco et al. (2006a) estimated a break at $150 \mathrm{~km} \mathrm{~s}^{-1}$, corresponding to $1.34 h^{-1} \mathrm{cMpc}$. We convert velocity differences into a redshiftpath length, $r=\Pi=(1+z) \Delta v / H(z)$, using the median redshift of $R R$ pairs in each bin. We assume a real-space correlation function of the form

$$
\xi(r)= \begin{cases}\left(r_{0} / r\right)^{\gamma} & \text { for } r \geqslant r_{1} \\ \left(r_{0} / r_{1}\right)^{\gamma} & \text { for } r<r_{1}\end{cases}
$$

The redshift-space correlation function follows from Equation (15) of Hawkins et al. (2003), which estimates the perturbation caused by linear infall. Due to the slope of the correlation function, infall lowers the amplitude of the line-ofsight correlation function at separations much larger than $r_{1}$. At smaller separations where the real-space correlation amplitude is constant, the volume effect caused by infall again boosts the correlation amplitude (as it did for the transverse correlation function).

The best fit to the joint ESI plus UVES data yields $r_{0}=$ $4.9 \pm 0.7$ and $r_{1}=1.2 \pm 0.3$ for $\gamma=1.6$. This correlation length and power-law index are consistent with the values $r_{0}=4.0 \pm 0.6 h^{-1}$ comoving Mpc and $\gamma=1.6 \pm 0.1$ fitted to the galaxy-galaxy correlation function at $z=2.9$ (Adelberger et al. 2005a). Fixing $r_{0}=4.0 h^{-1}$ comoving Mpc and $\gamma=1.6$, we fit $r_{1}=1.08 \pm 0.17 h^{-1}$ comoving Mpc to the joint ESI plus UVES line-of-sight $\mathrm{C}$ IV correlation function. This fit and the $1 \sigma$ uncertainty range are plotted in Figure 8 . They provide a reasonable description of the data. However, the correlation length $r_{0}$ strongly influences the fitted break at $r_{1}$; and we examine the implications of this parameter degeneracy in the next section.

\section{DISCUSSION}

On scales larger than the typical virialized halo, the correlation function of galaxies is expected to represent the distribution of dark matter halos that host them. A larger correlation length $r_{0}$ corresponds to a greater probability of finding a neighbor. Populations that cluster more strongly have higher $r_{0}$ and reside in more massive halos. From the cross-correlation of Lyman-break galaxies with $\mathrm{C}_{\text {IV }}$ systems, Adelberger et al. (2005b) showed that C IV systems with column density $\gtrsim 10^{13} \mathrm{~cm}^{-2}$ cluster as strongly as Lyman-break galaxies. It follows that these metals and Lyman-break galaxies populate the same halos. Tens of thousands of Lyman-break galaxies have been identified photometrically, and many thousand observed spectroscopically. It therefore seems reasonable to assume that the single power law that describes the clustering of Lyman-break galaxies on scales greater than $1 h^{-1}$ comoving Mpc also describes C IV systems on large scales. On scales smaller than virialized halos, multiple galaxies orbit within a deeper potential well; and the clustering of galaxies is harder to predict due to dissipational processes.

Our interest here is the scale of metal-enriched regions $r_{1}$. On scales $r<r_{1}$, the circulation of metals by feedback processes smoothes out their distribution; and the C IV auto-correlation amplitude is roughly constant. A difference between the fitted value of $r_{1}$ in redshift space and in real space is of particular interest. It may reflect the Doppler shifts of enriched regions.

For the C IV systems, the fitted $r_{1}$ depends on the correlation length. As $r_{0}$ increases, the clustering amplitude at any separation $r>r_{0}$ increases. To remain consistent with the measured correlation strength at small separations, the $r_{1}$ transition must shift to larger scales, where the correlation amplitude is lower. Figure 9 shows how the fitted $r_{1}$ grows with increasing correlation length. Based on the shape of the contours, the line-of-sight measurements clearly constrain the scale $r_{1}$ more tightly than they do the correlation length $r_{0}$. The difference in $r_{1}$ values and their implied Doppler shifts decrease as the correlation length increases.

The underlying real-space correlation length $r_{0}$ must describe both the transverse and line-of-sight clustering measurements. Clustering along the line of sight provides the better constraint because our sightlines to the binary quasars were simply not far enough apart to constrain $r_{0}$ well. Both measured C IV correlation functions, nonetheless, are marginally consistent with the Lyman-break galaxy correlation length, vertical line at $r_{0}=4.0 \pm 0.6 h^{-1}$ comoving Mpc in Figure 9. The bestfit correlation length $r_{0}=4.9 \pm 0.7 h^{-1}$ is slightly larger. In the absence of any peculiar velocities, the correlation length must be increased to $6 \mathrm{~h}^{-1}$ comoving Mpc before the same $r_{1}$ fits the clustering measured in both redshift space and real space. Our C IV observations provide only weak constraints on very large scale clustering, so they alone do not rule out $r_{0} \simeq$ $6 h^{-1}$ comoving Mpc. However, such halos are about three times more massive than those surrounding Lyman-break galaxies and have a number density about four times lower. ${ }^{15}$ In light of the strong cross-correlation amplitude between C IV systems and galaxies (Adelberger et al. 2005b) and the presence of outflows from most Lyman-break galaxies (Steidel et al. 2010), we do not discuss this possibility further.

For $r_{0}=4.0$ or $4.9 h^{-1}$ comoving Mpc, the implied peculiar velocities set an upper limit on the average speed of galactic

\footnotetext{
${ }^{15}$ At $z=2.0$, Lyman-break galaxies have $M_{h}=10^{11.2}-10^{11.8} M_{\odot}$ (Adelberger et al. 2005a).
} 


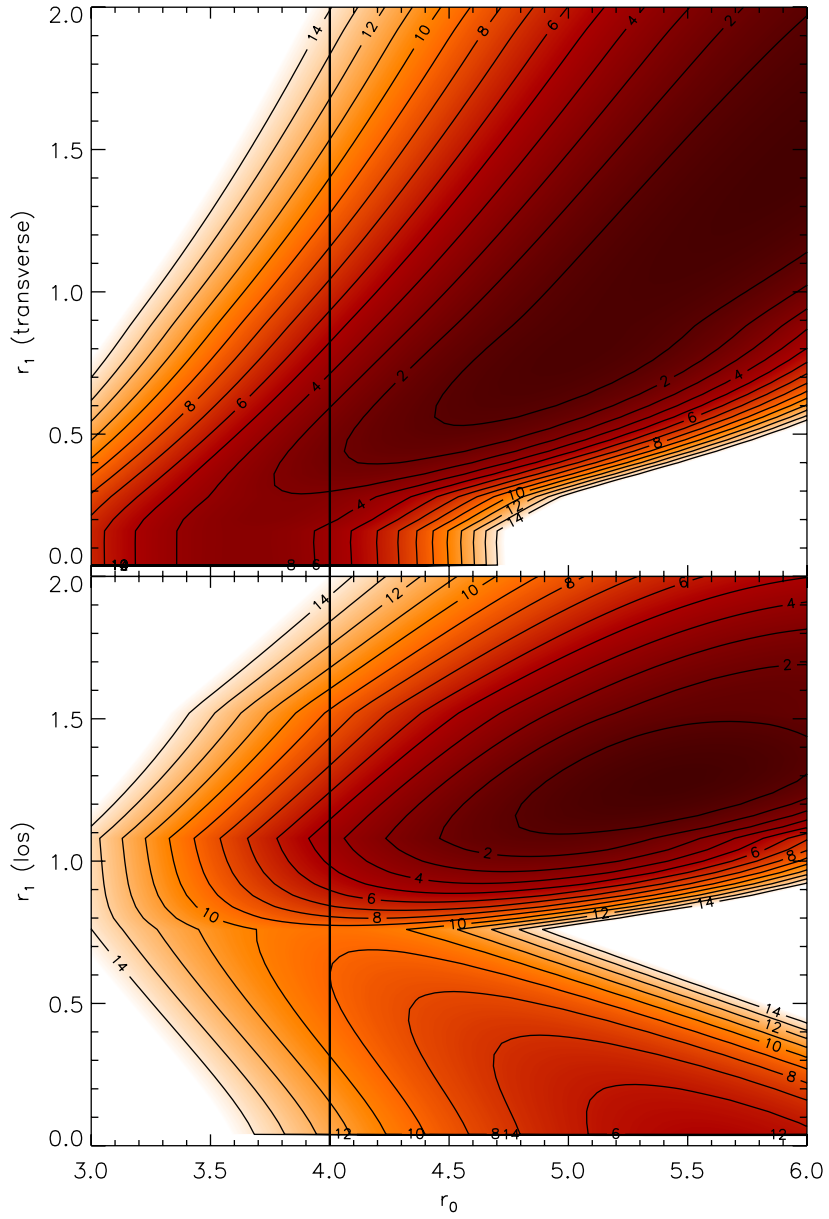

Figure 9. Contours of $\Delta \chi^{2}$ for two-parameter fits to the $\mathrm{C}$ IV correlation function. The correlation length $r_{0}$ and the size of enriched regions $r_{1}$ parameterize Equation (7), and the power-law index is taken to be $\gamma=1.6$. The fitted transverse and line-of-sight C IV correlation functions are illustrated separately in the upper and lower panels, respectively. The contours indicate values of $\Delta \chi^{2}$, where $2.30,4.61$, and 6.17 correspond to the $68.3 \%, 90.0 \%$, and $95.4 \%$ confidence level. The line-of-sight and transverse correlation functions sample the same enriched regions, so they must be jointly fit with the same $r_{0}$ and $r_{1}$. Adopting the clustering length of Lyman-break galaxies (vertical line) implies the presence of peculiar velocities among C IV systems. Larger values of the correlation length require smaller velocities. No Doppler shifts are required when the correlation length increases to (the rather large value of) $6 h^{-1}$ comoving Mpc and $r_{1} \simeq 1.3 h^{-1}$ comoving Mpc. (The fits to the line-ofsight clustering bifurcate at $r_{0} \gtrsim 4.5 h^{-1}$ comoving Mpc due to errors in counting $\mathrm{C}$ IV systems separated by the doublet spacing, $\Delta v=500 \mathrm{~km} \mathrm{~s}^{-1}$.)

(A color version of this figure is available in the online journal.)

outflows. Together, the size and speed measurements place interesting new constraints on the era of metal dispersal.

\subsection{The Size of Metal-enriched Regions}

\subsubsection{Amplitude of the Transverse Correlation Function}

As our best estimate for the size of metal-enriched regions, we adopt $r_{0}=4.0 h^{-1}$ comoving Mpc and $\gamma=1.6$ (Adelberger et al. 2005a). We fit the model given by Equation (7) to the transverse correlation function and find the power-law flattens on a scale $r_{1}=0.42 \pm 0.15 h^{-1}$ comoving Mpc. This fit is shown in the top panel of Figure 10, where it provides a good description of our measurements. For $r_{0}=4.0 \mathrm{~h}^{-1}$ comoving $\mathrm{Mpc}$, the fit to the line-of-sight correlation function shown in Figure 8 requires larger $r_{1}=1.08 \pm 0.17 h^{-1}$ comoving Mpc. A model illustrating this larger transition scale is shown in the

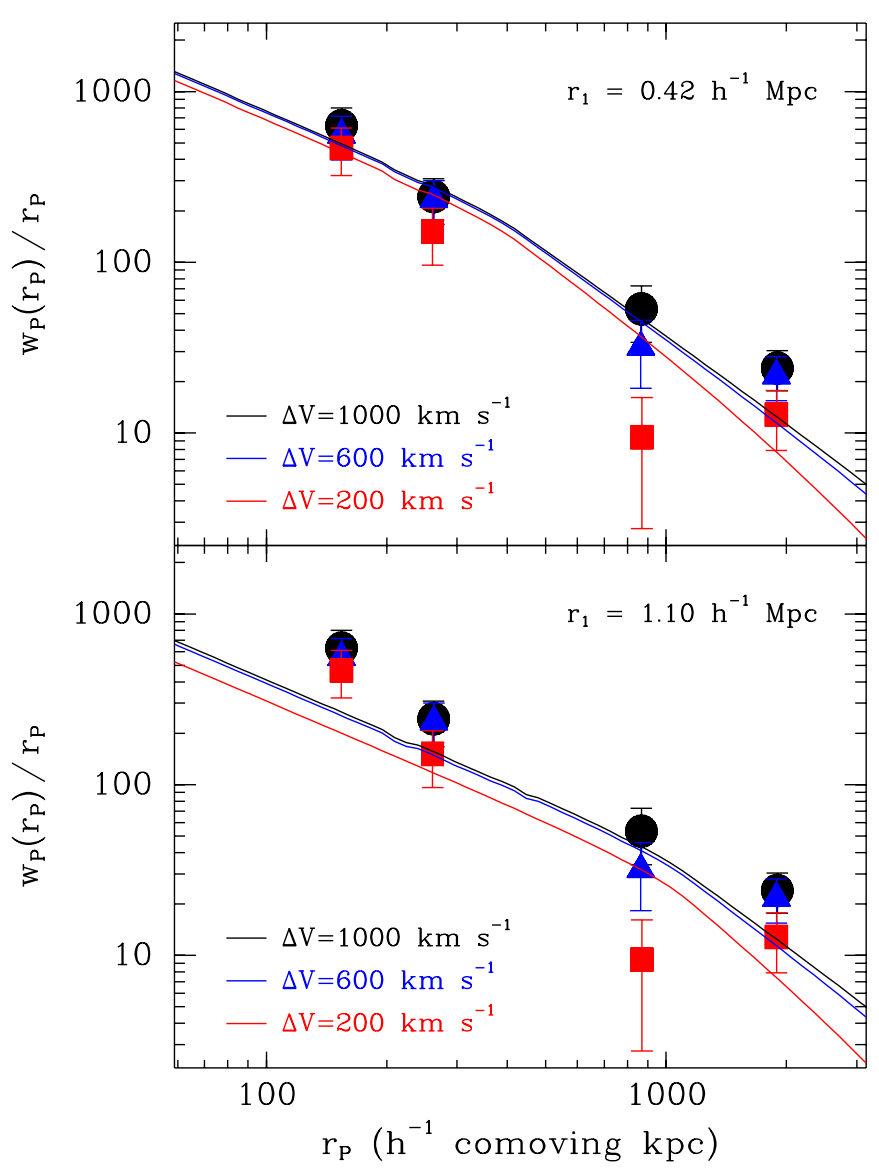

Figure 10. Model of the correlation function defined by Equation (7) in real space and then corrected for linear infall using Equation (15) of Hawkins et al (2003). We fix the correlation length at $r_{0}=4.0 \mathrm{~h}^{-1}$ comoving Mpc and powerlaw index at $\gamma=1.6$ (Adelberger et al. 2005a). We illustrate how the scale $r_{1}$ affects the transverse correlation amplitude. Data are as shown in Figure 7. Top: a fit to these data (specifically $\Delta v=100 \mathrm{~km} \mathrm{~s}^{-1}$ ) suggests $r_{1}=0.42 \pm$ $0.15 h^{-1}$ comoving Mpc. We show this model and interpret $r_{1}$ as the typical radius of an enriched region. Bottom: we show a model with $r_{1}=1.08 \mathrm{~h}^{-1}$ comoving Mpc, as favored by our fit to the line-of-sight correlation function. The larger size for the enriched regions provide too little power on small scales. (A color version of this figure is available in the online journal.)

bottom panel of Figure 10, where it clearly underpredicts the correlation between sightlines seen on small scales. We find $\mathrm{C}$ IV systems at similar redshifts in the two sightlines more often than we would in a model with metal bubbles as large as those suggested by Scannapieco et al. (2006a). Consistency requires a model with redshift-space distortions.

We also illustrate the discrepancy in redshift space when the $\mathrm{C}$ IV correlation length takes our best-fit value of $r_{0}=$ $4.9 h^{-1}$ comoving Mpc for $\gamma=1.6$. We fitted $r_{1}=1.21 \pm$ $0.25 h^{-1}$ comoving Mpc to the line-of-sight correlation function. To the transverse measurement, we fitted $r_{1}=0.74 \pm$ $0.21 h^{-1}$ comoving Mpc. The scale of the line-of-sight break remains significantly larger than the fitted transverse break. Evidently, the metal-enriched regions present peculiar velocities with respect to the cosmic expansion. We fit the magnitude of these velocity kicks in Section 4.2.

\subsubsection{Coherence Length from Coincident Absorption in Spectra of Binary Quasars}

The spectra of binary or lensed quasars have been used previously to constrain the transverse size of absorbing structures. The transverse size of a coherent region was inferred to be at 
least as large as the maximum separation of common spectral features (McGill 1990; Lopez et al. 2000; Ellison et al. 2004). As implemented previously, however, the resulting coherence length has little to do with the size of enriched regions. To illustrate this important point, we apply the standard coherencelength analysis to our sample of C IV systems, explain why it fails, and suggest a revised technique. This revised coherencelength estimator can be compared to the size scale $r_{1}=0.42$ or $0.74 h^{-1}$ comoving Mpc fitted to the transverse correlation function.

To estimate the coherence scale of C IV absorption, we first follow the formalism of McGill (1990) with the modification suggested by Dinshaw et al. (1997). Their argument is entirely geometric in nature. They describe absorption-line systems with a non-evolving population of clouds. For illustration, we adopt a spherical distribution of clouds, but the method easily generalizes to cylindrical filaments or inclined disks. The radius $R$ describes the coherence scale of the cloud distribution. For pairs of sightlines separated by a distance $l,{ }^{16}$ the probability that the second sightline intersects the sphere, given that the first sightline "hit" the sphere is

$$
P_{1}=2 / \pi\left\{\cos ^{-1} X(z)-X(z) \sqrt{1-X(z)^{2}}\right\},
$$

where $X(z)=l(z) /(2 R)$ and $0 \leqslant X(z) \leqslant 1$. If either sightline shows absorption, the probability of coincident absorption, defined as C IV absorption in both sightlines at similar redshift, is

$$
P_{2}=P_{1} /\left(2-P_{1}\right) \text {. }
$$

The probability of absorption in just one sightline, an anticoincidence, is $1-P_{2}$. For a particular coherence length $R$, the likelihood of obtaining the observed number of coincident systems and the observed number of anti-coincidences is

$$
\mathcal{L}(R)=\Pi_{i=1}^{N_{i}} P_{2}\left[X\left(z_{i}\right)\right] \Pi_{j=1}^{N_{j}}\left(1-P_{2}\left[X\left(z_{j}\right)\right]\right),
$$

where the two products are over all coincidences $N_{i}$ and anticoincidences $N_{j}$, respectively. Figure 11 shows the likelihood for many different values of the coherence length.

Applied to the entire sample of 29 binary quasars, the maximum likelihood is found at the very large scale of $R=1.07_{-0.02}^{+0.07}$ $h^{-1}$ comoving Mpc. Detection of just a single coincidence between sightlines separated by $l=2.1 h^{-1}$ comoving Mpc sets a sharp lower bound. In general, the analysis proposed by McGill (1990) and Dinshaw et al. (1997) requires the sphere diameter to be at least as large as the separation of the widest pair of sightlines with a coincidence. As we argued at the beginning of Section 4, however, the sources of metals are highly clustered. When two sightlines are separated by $\sim 1 h^{-1}$ comoving Mpc, the correlation between the halos of their sources causes some absorption at a common redshift. The clustering of galaxies, and therefore metal-enriched regions, has not been accounted for in Equation (8). This oversight leads to a coherence scale that grows with the separation of the widest binary quasar in the sample. Figure 11 demonstrates this lack of convergence by comparing the likelihood functions for three subsets of data.

In principle, it might be possible to modify the analysis to obtain a robust coherence length for enriched regions. This

\footnotetext{
16 Although previous authors used the physical separation of the sightlines, the comoving separation must be used when the absorber redshifts span a significant range in the growth factor, $1+z$.
}

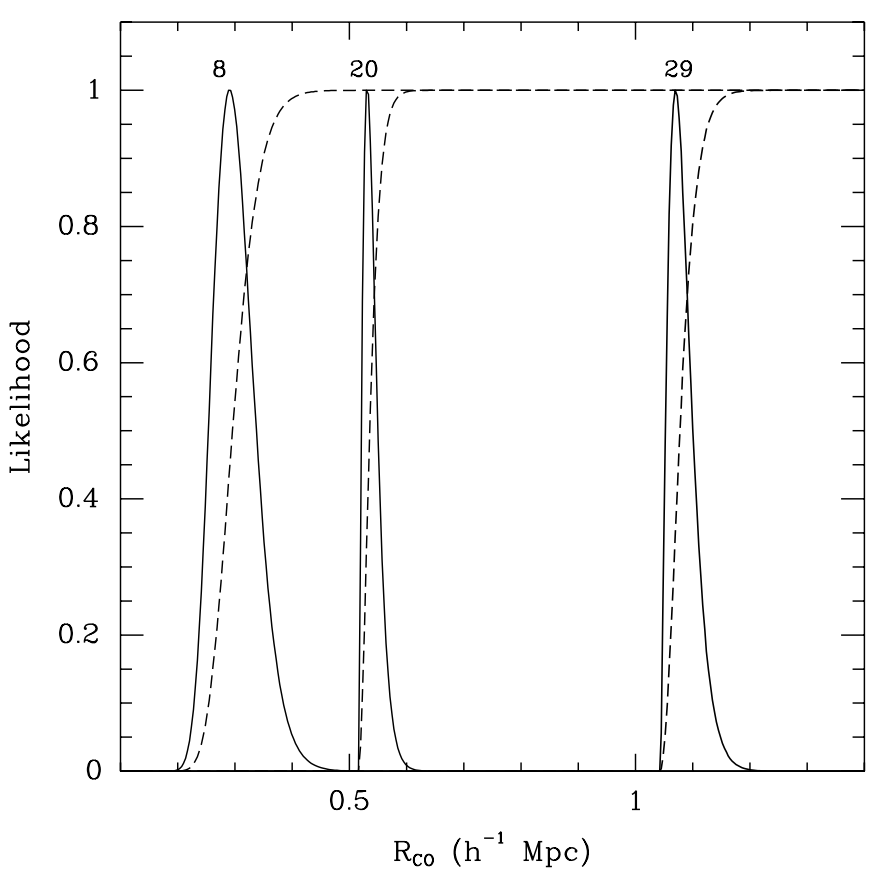

Figure 11. Likelihood of observed coincident and anti-coincident C IV systems (normalized by the maximum likelihood) vs. the coherence length of a spherical distribution of clouds. The dashed line shows the cumulative distribution, which we use to define the $95 \%$ confidence limit on the statistical error. We defined coincidences as systems in different sightlines within $600 \mathrm{~km} \mathrm{~s}^{-1}$ of each other. The most-likely coherence radius $R=1.07_{-0.02}^{+0.07} h^{-1} \mathrm{Mpc}$ for all 29 pairs. However, the best radius drops to $R \simeq 0.5 h^{-1} \mathrm{Mpc}$ when the nine widest binaries are dropped from the sample; and $R \simeq 0.3 h^{-1} \mathrm{Mpc}$ when the analysis is applied to only the closest eight binaries in the sample. As sample size increases (and wider binaries are included), the maximum-likelihood analysis does not converge to a coherence length. See the text for explanation.

provides no obvious advantage over the $\mathrm{C}$ IV correlation function but offers a sanity check on the inferences drawn from the correlation function. We suggest that the maximum-likelihood estimate of the coherence length may converge provided the sightline separations remain smaller than the typical size of individual enriched regions. When the likelihood analysis yields $R$ comparable to one-half the maximum sightline separation, the method has not converged. To estimate this coherence scale, we calculated the function given by Equation (10) for a series of 1, $2,3, \ldots$, and 29 binary quasars. Figure 12 compares our result to the line $2 R=l$. When the sightline separation is smaller than the diameter of the typical enriched region, $l<2 R$, we expect many coincidences, few anti-coincidences, and a most-likely coherence diameter $2 R$ significantly larger than the maximum sightline separation.

In Figure 12, the coherence-length estimate for all subsamples with maximum sightline separation less than $0.6 h^{-1}$ comoving Mpc allows $R \approx 0.3 h^{-1}$ comoving Mpc. In contrast, as the maximum sightline separation grows larger than $0.6 h^{-1}$ comoving $\mathrm{Mpc}$, the estimated coherence length follows the locus $R=0.5 l$. The coherence length appears to converge to a value $R \approx 0.3 h^{-1}$ comoving Mpc in Figure 12 . This scale is consistent with the $0.42 \pm 0.15 h^{-1}$ comoving Mpc fitted to the transverse correlation function with $r_{0}=4.0 h^{-1}$ comoving Mpc. It is marginally inconsistent with the larger $r_{1}$ fitted when a larger correlation length is adopted. Given the many implicit assumptions in the interpretation of both $R$ and $r_{1}$, and the acknowledgement that enriched regions could have a range of sizes, we believe the revised coherence-length analysis 


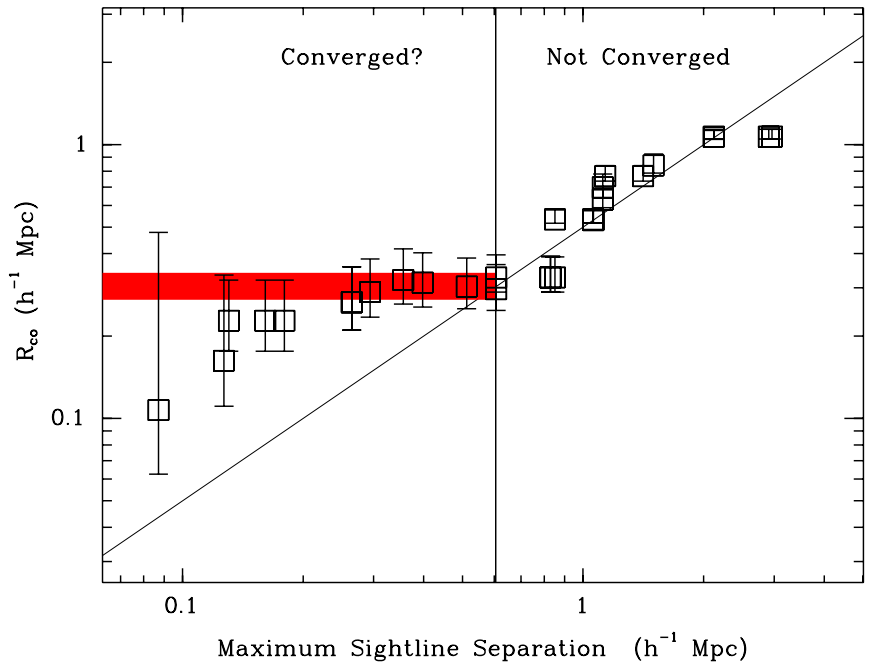

Figure 12. Coherence length (in comoving units) for different subsets of paired sightlines. Using our large set of pair observations at varying separations, we iteratively threw out the largest pair and recomputed the coherence length using the maximum-likelihood analysis. The $x$-axis indicates the widest (comoving) separation included in the subsample. The diagonal line indicates a sphere radius of one-half the maximum sightline separation, $R=0.5 l$. The estimated coherence length does not converge as the sample size is increased. When $R$ is significantly greater than $0.5 l$, however, this method may provide a robust estimate of the coherence length (shaded, red) of individual enriched regions.

(A color version of this figure is available in the online journal.)

lends strong support to our proposed interpretation of the C IV correlation function. It may rule out $r_{1}$ as large as $0.7 h^{-1}$ comoving Mpc, but we prefer to see the technique calibrated against cosmological models before making this claim.

\subsubsection{Previous Observations of C IV Absorption near Lyman-break Galaxies}

Sightlines passing by known Lyman-break galaxies directly probe the properties of circum-galactic gas. Spectra of background light beacons present intervening absorption near the redshift of Lyman-break galaxies. Because background galaxies are numerous, spectroscopy of galaxies can probe circumgalactic gas at low impact parameter. Background quasars are less common, but spectra of the brightest quasars provide higher sensitivity to absorption lines. The impact parameters of these detections can be compared to our measured scale for the enriched regions, about 150 physical kpc.

Composite galaxy spectra were recently created over a range of impact parameters, $b$, from Lyman-break galaxies (Steidel et al. 2010). Intervening $C$ IV absorption was detected at $b=60$ physical kpc but not at $b=100 \mathrm{kpc}$. Our analysis suggests the radius of the typical metal-enriched region is significantly larger. Is this a sensitivity difference or a discrepancy?

Our sample contains C IV systems as strong as the $W_{r, 1549}=$ $700 \mathrm{~m} \AA$ absorption trough in the composite spectrum at $b=$ $60 \mathrm{kpc}$. Our spectra of median quality are more sensitive than their detection threshold of $W_{r, 1549}=120 \mathrm{~m} \AA$ at $b=$ $100 \mathrm{kpc}$. Figure 3 illustrates our equivalent-width distribution. The typical detection limit is $W_{r, 1551}=0.5 W_{r, 1549} \simeq 20 \mathrm{~m} \AA$; and a few spectra reach $W_{r, 1551}=0.5 W_{r, 1549} \simeq 8 \mathrm{~m} \AA$. Our sensitivity to weaker systems may explain why we find a larger size for the enriched regions.

These weaker C IV systems do appear to be associated with the environment of Lyman-break galaxies. The clustering of weaker systems has been measured using sensitive spectra of
23 quasars in Lyman-break galaxy survey fields. The crosscorrelation length (between Lyman-break galaxies and C IV systems) increases slowly with the C IV column density (Adelberger et al. 2005b, Figure 11). At the completeness limit of our survey, $W_{r, 1551} \simeq 20 \mathrm{~m} \AA$, C IV systems cluster as strongly with Lyman-break galaxies as Lyman-break galaxies do with each other. Hence, we can be reasonably confident that the large metal-enriched regions describe the environment of Lymanbreak galaxies. We have not shown, nor do we claim, that these weaker C IV systems mark outflows from Lyman-break galaxies.

\subsection{The Peculiar Velocities of Metal-enriched Regions}

It is clearly of interest to understand why the line-of-sight correlation between CIV systems flattens on a larger scale than does their transverse correlation function. The line-ofsight correlation function is derived from the redshift difference of pairs of C IV systems. Their separation in redshift mixes information about their physical separation and their relative Doppler shifts. Non-zero peculiar velocities smear out the correlation in redshift space. Adding random velocity kicks to enriched regions therefore flattens the line-of-sight correlation function on a scale somewhat larger than $r_{1}$. We can fit the magnitude of the typical velocity kick.

As suggested by Hawkins et al. (2003), we represent the random motions by an exponential of the form

$$
f(v)=\frac{1}{a \sqrt{2}} \exp \left(-\frac{\sqrt{2}|v|}{a}\right) .
$$

The impact of the Doppler velocity $a$ on the line-of-sight correlation function is shown in Figure 13, where these kicks increase from zero to $a=1000 \mathrm{~km} \mathrm{~s}^{-1}$. The correlation amplitude decreases at velocity separations $\Delta v<a$ and grows on the scale $\Delta v \simeq a$. The magnitude of the velocity kick required to fit the correlation function depends on $r_{1}, r_{0}$, and $\gamma$. The largest value of $a$ results if we adopt the smallest value of $r_{1}$ (and therefore the smallest correlation length). For example, taking $r_{1}=0.42 h^{-1}$ comoving Mpc as shown in the top panel of Figure 13, we fitted $a \simeq 120 \mathrm{~km} \mathrm{~s}^{-1}$-the model shown by the bold line. With no velocity kick, we fitted a larger $r_{1}=1.08$ $h^{-1}$ comoving Mpc to these data in Figure 8. Models with these larger enriched regions do not allow any velocity kicks. As illustrated in the bottom panel of Figure 13, large enriched regions with peculiar velocities yield too few pairs of C IV systems at separations of a few hundred $\mathrm{km} \mathrm{s}^{-1}$. To summarize, we quantify the upper limit on $a$ in Figure 14. Varying the Doppler velocity and computing the fit statistic, we find a $3 \sigma$ upper limit $a \leqslant 300 \mathrm{~km} \mathrm{~s}^{-1}$. We conclude that the average Doppler shift of metal-enriched regions along the line of sight is likely about $120 \mathrm{~km} \mathrm{~s}^{-1}$ and appears very unlikely to exceed $300 \mathrm{~km} \mathrm{~s}^{-1}$.

The average peculiar velocity of metal-enriched regions in three-dimensions is $v_{\mathrm{pec}}=\sqrt{3} a \simeq 200 \mathrm{~km} \mathrm{~s}^{-1}$ ( $3 \sigma$ upper limit of $500 \mathrm{~km} \mathrm{~s}^{-1}$ ) after correction for projection along the line of sight. These peculiar velocities are similar to those of galaxies. Their primary source may be gravitational instability associated with the underlying mass structure. The outflow speed measured in galaxy spectra is $\simeq 200 \mathrm{~km} \mathrm{~s}^{-1}$ (Martin 2005; Weiner et al. 2009; Steidel et al. 2010). This absorbing gas lies close the host galaxy (Martin \& Bouché 2009). The average outflow speed, where the average is a spatial average from the starburst region out to the bubble radius where the wind stalls or 

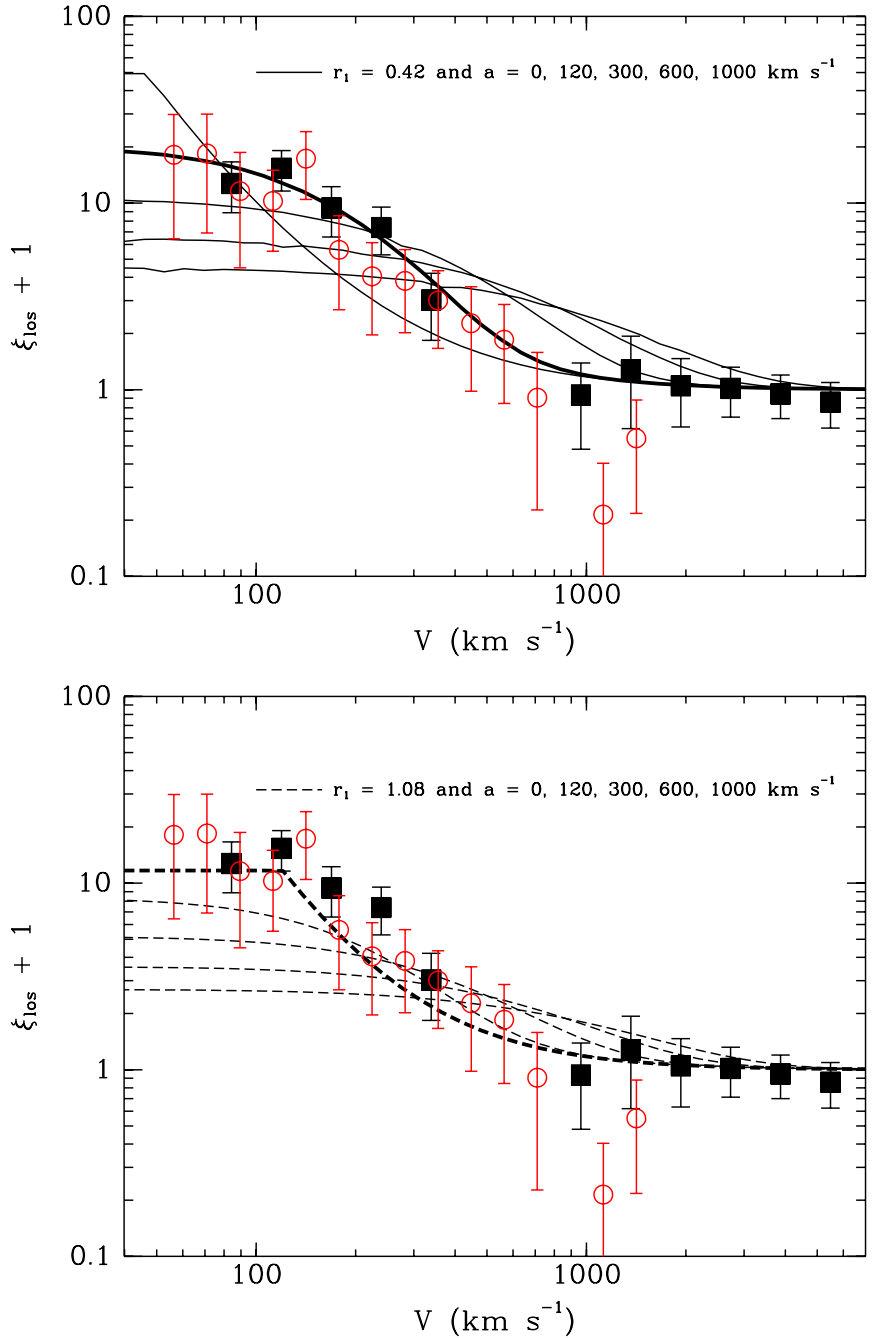

Figure 13. Models for the line-of-sight correlation function. The parameter $a$ in Equation (11) describes the typical random velocity of an enriched region. The real-space correlation function and infall models are the same as those shown in Figure 10 (with $r_{0}=4.0 h^{-1}$ comoving Mpc and $\gamma=1.6$ ). Data are as in Figure 8 with the bins affected by the doublet spacing omitted for clarity. Top: we fix $r_{1}=0.42 h^{-1}$ comoving Mpc, the value favored by our fit to the transverse correlation function. Thick line shows the best-fit peculiar velocity $a=120 \mathrm{~km} \mathrm{~s}^{-1}$. Thin lines illustrate other peculiar velocities. Lower velocities give too much correlation on small scales and higher velocities require more pairs at velocity separations $\Delta v \approx 300-1000 \mathrm{~km} \mathrm{~s}^{-1}$ than are observed. Bottom: here, we fix $r_{1}=1.08 h^{-1}$ comoving Mpc, the fitted bubble size for no peculiar velocity (bold line). We show that adding even a small peculiar velocity of $a=120 \mathrm{~km} \mathrm{~s}^{-1}$ results in too few pairs of C IV systems at $\Delta v<a$ and too many pairs at $\Delta v>a$.

(A color version of this figure is available in the online journal.)

even turns around, has not been directly measured previously. Our measurement shows that this average outflow speed is very unlikely to be larger than $200 \mathrm{~km} \mathrm{~s}^{-1}$. The average velocity may be considerably less because gravitationally induced peculiar velocities must contribute to random velocity kicks.

Adelberger et al. (2005b) reached a very different conclusion about average outflow speeds. Their Figure 12 shows the distribution of redshift differences between C IV systems and Lyman-break galaxies. If the galaxy and gas are co-spatial, the typical offset in redshift corresponds to a Doppler shift of $\simeq 500 \mathrm{~km} \mathrm{~s}^{-1}$. Because such velocities are larger than virial motions within Lyman-break galaxies, this observation was interpreted as evidence for galactic outflows at $500 \mathrm{~km} \mathrm{~s}^{-1}$ or more.

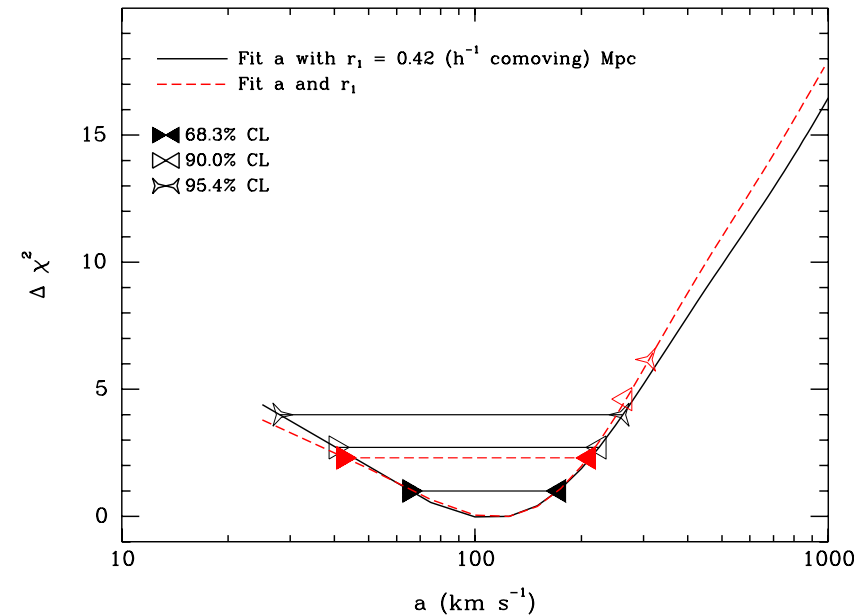

Figure 14. Values of peculiar velocity $a$ fitted to the line-of-sight $\mathrm{C}$ IV correlation function. With $r_{1}$ fixed at $0.42 h^{-1}$ comoving Mpc (solid line), the deviation in $\chi^{2}$ from the best fit allows peculiar velocities up to 170,220, and $260 \mathrm{~km} \mathrm{~s}^{-1}$ at the $1 \sigma, 2 \sigma$, and $3 \sigma$ confidence level. A more general twoparameter fit to $a$ and $r_{1}$ simultaneously (dashed line) allows a maximum peculiar velocity of $310 \mathrm{~km} \mathrm{~s}^{-1}$ at the $3 \sigma$ level. (The correlation function of Adelberger et al. 2005a is assumed for $r_{0}$ and $\gamma$.)

(A color version of this figure is available in the online journal.)

Outflow speeds of $500 \mathrm{~km} \mathrm{~s}^{-1}$ are inconsistent with the measured line-of-sight correlation function for C IV systems. We suggest that the redshift differences between C IV systems and Lyman-break galaxies can be understood without appealing to fast outflows. The physical separation between the absorbing gas and the galaxy may explain their redshift differences. For example, cosmic expansion at $z \simeq 3$ produces a velocity difference of $450 \mathrm{~km} \mathrm{~s}^{-1}$ over the $4.0 \mathrm{~h}^{-1}$ comoving Mpc (the correlation length). As the column density of C IV systems increases, their cross-correlation length (with galaxies) increases. For larger cross-correlation length, which is equivalent to a stronger correlation amplitude, the distance from a galaxy to the nearest intervening systems is smaller. The average column density of the absorption will be higher the smaller the separation or impact parameter. The strongest $\mathrm{C}$ IV systems are always found at small impact parameters, so random spatial offsets between galaxies and C IV systems can plausibly explain the increase in redshift differences observed with growing impact parameter.

\subsection{The Dispersal of Metals}

Understanding how C IV systems cluster reshapes our view of the IGM enrichment history. In particular, the large sizes and low peculiar velocities of enriched regions constrain the era of metal dispersal. This process is of course ongoing as there is strong observational evidence for galactic winds across much of cosmic time. Not all galaxy populations, however, can easily distribute metals as widely as observed.

\subsubsection{Lyman-break Galaxies at $z \simeq 3$}

How far can a galactic wind from an individual galaxy can transport metals? In the absence of any forces to stall the expansion, the maximum distance traveled is limited by the age of the starburst galaxy and average velocity. Adopting the upper limit $\langle v\rangle \simeq 200 \mathrm{~km} \mathrm{~s}^{-1}$ compatible with line-of-sight clustering observations, galactic winds travel

$$
R \simeq 61 \mathrm{kpc}\left(\frac{v}{200 \mathrm{~km} \mathrm{~s}^{-1}}\right)\left(\frac{\tau}{300 \mathrm{Myr}}\right)
$$


in a typical Lyman-break galaxy lifetime. ${ }^{17}$ This distance is at most $40 \%$ of our estimated radius for an enriched region. While typical Lyman-break galaxies clearly eject metals in winds, other galaxies may spread metals over larger regions of intergalactic space.

The properties of Lyman-break galaxies do span a significant range; and the winds from those with the largest lifetimes can enrich the large regions we find. To disperse metals out to $200 \mathrm{kpc}$, the required age is $\sim 1 \mathrm{Gyr}$ for an average speed $\simeq 200$ $\mathrm{km} \mathrm{s}^{-1}$. Ages $\sim 1$ Gyr are fit to about $20 \%$ of Lyman-break galaxies (Shapley et al. 2001). Interestingly, the Lyman-break galaxies with older ages tend to be less luminous (Shapley et al. 2001). It follows that the lowest luminosity Lyman-break galaxies (in current samples) could be the source of the most dispersed metals.

Given the absorption cross section of bubbles with $r_{1} \simeq$ $0.42 h^{-1}$ comoving Mpc, the Lyman-break galaxies are not numerous enough to be the source of most of the C IV systems. The number density of Lyman-break galaxies is $n_{g} \approx 3.7 \times$ $10^{-3} h^{3} \mathrm{Mpc}^{-3}$ (Steidel et al. 2010). If we could place these bubbles around every Lyman-break galaxy, the resulting redshiftpath density of C IV systems would be about

$$
\begin{aligned}
\frac{d N}{d z} \approx & 1.4\left(\frac{n_{\mathrm{gal}}}{3.7 \times 10^{-3} h^{3} \mathrm{Mpc}^{-3}}\right) \\
& \times\left(\frac{r_{1}}{0.42 h^{-1} \mathrm{Mpc}}\right)^{2}\left(\frac{450 h \mathrm{~km} \mathrm{~s}^{-1} \mathrm{Mpc}^{-1}}{H(z)}\right) .
\end{aligned}
$$

For comparison, integration of our distribution function shown in Figure 3 to $W_{r, 1551} \simeq 20 \mathrm{~m} \AA$ yields about $14, \mathrm{C}_{\text {IV }}$ systems per unit redshift. Each bubble of metals might yield more than one $\mathrm{C}$ IV system, but $\mathrm{C}$ IV systems outnumber Lymanbreak galaxies by an order of magnitude.

\subsubsection{Post-reionization Dispersal of Metals}

Large bubbles of metals surrounding Lyman-break galaxies could be relics of an era when galaxies were smaller, more numerous, and closer together (Porciani \& Madau 2005; Scannapieco 2005). How do the new results on the clustering, size, and peculiar velocities of enriched regions fit into this picture?

In order for their remnant winds to cluster like C IV systems, higher-redshift sources must have lower mass. Consider the post-reionization galaxy population, for example. Redshift 6 galaxies in halos about 30 times less massive than those of Lyman-break galaxies have the same bias as the Lyman-break galaxies at $z \simeq 3$. At redshift 4.3 galaxies five times less massive than Lyman-break galaxies would eject metals that clustered like Lyman-break galaxies by $z \simeq 3$.

Low-mass galaxies naturally produce large enriched regions. In a shallow gravitational potential, gas retainment is more difficult. Because enrichment is earlier, the bubbles around lowmass galaxies have more time to grow. At an average speed of $\simeq 200 \mathrm{~km} \mathrm{~s}^{-1}$, the bubble could travel 150 physical kpc between

\footnotetext{
17 For purposes of illustration, we use the median timescale for star formation, $321 \mathrm{Myr}$, from Table 4 of Shapley et al. (2001). With only optical photometry, the absolute ages are quite sensitive to the model for the star formation history. For example, Sawicki \& Yee (1998) fit much younger ages $\sim 30$ Myr. As shown by Shapley et al. (2001), measurement of the Balmer break distinguishes these interpretations and favors typical ages of a few hundred Myr.
}

redshift 4.3 and 3 . If growth stalls relative to the surrounding medium, the bubbles continue to grow in physical size due to cosmic expansion. (For a given physical size, the comoving bubble scale is larger the earlier the enrichment.) Most importantly, however, the number density of halos (and presumably galaxies) grows rapidly with decreasing mass. Those low-mass galaxies between $z=4.3$ and 6 that cluster like Lyman-break galaxies could easily be 5-30 times more numerous.

In the post-reionization universe, the potentially high number of galactic winds and large cross section for absorption yield a large density of metal-line systems. From Equation (13), the number per unit redshift is about 5 when starburst galaxies at redshift 4.3 are the source of metals observed at redshift 3 . The redshift-path density grows rapidly for earlier dispersal. The spatial distribution of the entire population of C IV systems with $N(\mathrm{C}$ IV $) \gtrsim 10^{13} \mathrm{~cm}^{-2}$ can easily be attributed to their relic galactic winds. Significant metal enrichment will of course continue until much later, but the post-reionization galaxies appear to dictate the size scale of the CGM.

The bubbles from numerous low-mass galaxies may overlap. Bubble coalescence would generate rapid growth in the size of the enriched regions. Such growth is demonstrated by simple analytic models. In Figure 2 of Scannapieco (2005), the comoving size of enriched regions grows slowly when rare galaxies provide the metals. Indeed, the bubbles blown by different Lyman-break galaxies never overlap. The probability of finding a second galaxy within the bubble volume $V$ is $P=n_{\text {gal }} V\left(1+\xi\left(r_{1}\right)\right)$. Substituting typical values, we estimate

$$
\begin{aligned}
P= & 0.04\left(\frac{n_{\mathrm{gal}}}{3.7 \times 10^{-3} h^{3} \mathrm{Mpc}^{-3}}\right) \\
& \times\left(\frac{r_{1}}{0.42 h^{-1} \mathrm{Mpc}}\right)^{3}\left(\frac{1+\xi\left(r_{1}\right)}{1+37}\right),
\end{aligned}
$$

where we adopt a correlation length of $4.0 \mathrm{~h}^{-1}$ comoving Mpc and $\gamma=1.6$ to estimate the probability of finding another Lyman-break galaxy within $r_{1} h^{-1}$ of the first. Since the bubbles blown by a Lyman-break galaxy with median age are not this large, this parameterization gives an upper limit on the odds of overlap. The $z \simeq 4.3$ starbursts are the youngest population (750 Myr at $z \simeq 3$ ) that have time to blow the large bubbles we found. Their five times higher source density yields $P \approx 0.2$ suggesting that many bubbles might remain isolated although approaching overlap. In contrast, relic winds from redshift 6 sources would overlap create enriched regions much larger than the bubbles blown by individual galaxies.

In summary, the measured size $r_{1}$ of the enriched regions is difficult to explain by metal dispersal within just a few hundred Myr of $z \simeq 3$. This observation does not distinguish slightly earlier enrichment at $z \simeq 4.3$ from enrichment at the end of cosmic reionization at $z \approx 6$. Enrichment over this era, however, might be distinguished from the Population III scenario by measuring rapid growth in the size of enriched regions. Population III stars in $1000 \mathrm{~K}$ halos would produce rapid growth in the comoving size of the enriched regions prior to redshift 6 . Post-reionization wind bubbles, on the other hand, may coalesce between redshift 6 and 3, generating rapid growth in the size of enriched regions. Our measured size, although large, does not require coalescence. Starbursts at redshifts 4-5 could create such bubbles, which would not have merged for the most part by $z \simeq 3$. Some of the parent galaxies would still be observable at redshift 3 and could be identified by their older stellar population and low mass. 


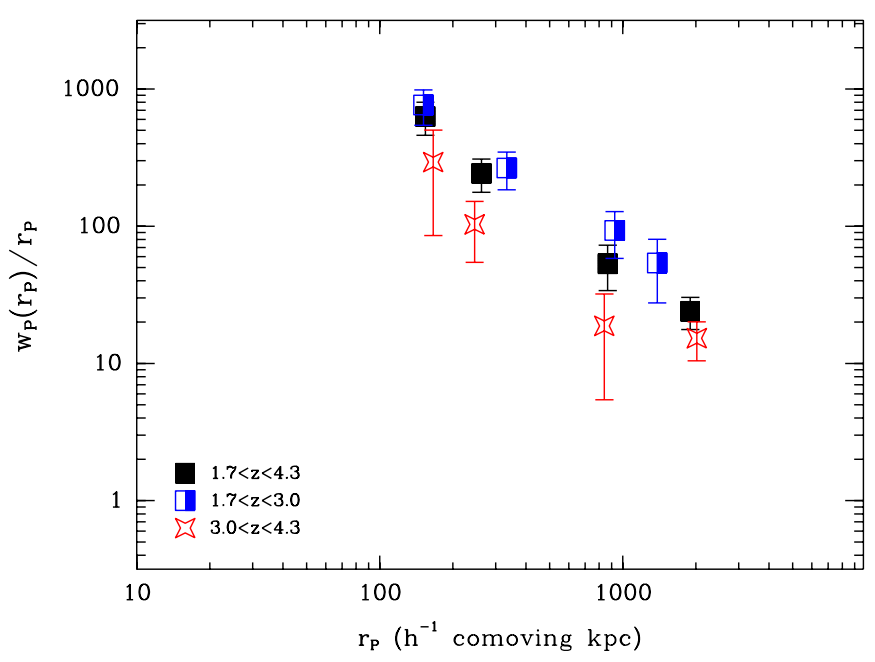

Figure 15. Transverse correlation function, as in Figure 7 with $\Delta v \leqslant$ $1000 \mathrm{~km} \mathrm{~s}^{-1}$, but for high- and low-redshift $\mathrm{C}$ IV systems. The correlation amplitude for the lower-redshift subsample at $z=2.46$ (blue, half-filled symbols) exceeds that measured for higher-redshift subsample at $z=3.33$ (red, starred symbols).

(A color version of this figure is available in the online journal.)

\subsection{Evolution of the Size of Enriched Regions}

The evolution of the CIV auto-correlation function may provide a useful constraint on the metal enrichment history. The size of enriched regions can only grow in comoving units if bubbles merge or additional enrichment takes place. As bubbles coalesce due to galactic infall, the size of the enriched region grows as gravity assembles galaxies into large-scale structures. The correlation amplitude for galaxy halos grows as $(1+z)^{-2}$. Evolution in the C IV correlation function inconsistent with $(1+z)^{-2}$ would indicate the addition or removal of metals.

Scannapieco et al. (2006a) found that the amplitude of the line-of-sight correlation functions for $\mathrm{Mg}$ II and Fe II, and possibly $\mathrm{C}$ IV, increases with cosmic time. We used our median redshift to define lower-redshift $C$ IV systems $\left(1.7<z_{a b} \leqslant 3\right)$ and higher-redshift systems $\left(3 \leqslant z_{a b}<4.3\right)$. For each subsample, we counted $D_{A} D_{A}, D_{A} R_{A}$, and $R_{A} R_{A}$ pairs in a single velocity bin at $50 \mathrm{~km} \mathrm{~s}^{-1}<\Delta v<500 \mathrm{~km} \mathrm{~s}^{-1}$. The median pair separation is about $\Delta v \approx 150 \mathrm{~km} \mathrm{~s}^{-1}$ at both redshifts. The correlation amplitude of the higher-redshift subsample is $\xi+1 \approx 5.5$. The amplitude grows to $\xi+1 \approx 11$ for lower-redshift subsample. Hence, we confirm the amplitude of $\xi_{\mathrm{LOS}}(\mathrm{C} \mathrm{IV})$, increases with cosmic time.

When we compare two sightlines, we count pairs with separations up to $\Delta v_{\text {pair }}=1000 \mathrm{~km} \mathrm{~s}^{-1}$. The identification of systems in different spectra is not biased by the $\mathrm{C}$ IV doublet spacing, and the larger velocity difference reduces the noise. In the higher-redshift subsample, the median redshift of an $R_{A} R_{B}$ pair is 3.33. The median pair redshift is 2.46 in the lowerredshift subsample. We calculate the transverse correlation function as described in Section 3.1.2 and show the result in Figure 15. At all sightline separations, the amplitude for the lower-redshift C IV systems is larger than that for the higherredshift systems. The magnitude of the increase in clustering strength is consistent with that measured along the line of sight. We estimate $\xi(2.46) / \xi(3.33) \approx 2$ for $\mathrm{C}$ IV systems

Between the median subsample redshifts of 3.33 and 2.46, we expect the amplitude of the correlation to grow by a factor 1.6. Our measured correlation functions for the subsamples are consistent with this growth rate, although the large error bars allow somewhat faster or slower growth. Measuring evolution in the scale of the break $r_{1}$ may be possible in the future. With the current sample, the uncertainties in the fitted $r_{1}$ are large and consistent with no evolution in $r_{1}$ larger than a factor of 2 . The addition of larger separation pairs to our analysis is important to allow fitting $r_{0}$ and $r_{1}$ without additional priors.

\section{SUMMARY}

We obtained echellete spectra of binary quasars, which probe the IGM along parallel sightlines. Metal-enriched regions were identified by $\mathrm{C}$ IV absorption systems. We measured their clustering in real space, transverse to the sightlines, and in redshift space, along individual sightlines. At large separations, the clustering of intervening $\mathrm{C}$ IV systems is consistent with the spatial distribution of galaxies. (Galaxies, as used here, referred to Lyman-break galaxies at $z \sim 2.9$ with correlation length $r_{0}=4.0 \mathrm{~h}^{-1}$ comoving Mpc.) We found metals more smoothly distributed than galaxies, however, below a scale that we define as the enriched region. This scale may represent the turnaround radii of galactic winds or the overlap of multiple bubbles. We showed that fast outflows would smear out the $\mathrm{C}$ IV correlation function in redshift space. By comparing the correlation functions in redshift space and real space, we found the maximum root-mean-square outflow velocity. We argued that the large size of the enriched regions and slow outflow speeds are incompatible with dispersal by winds from $z \simeq$ Lyman-break galaxies.

We list our empirical results here before discussing their implications.

1. We measured redshifts and equivalent widths for 316 intervening $\mathrm{C}$ IV systems at $1.7<z<4.3$. The number of systems per unit redshift path falls as the strength of the absorption increases. The distribution of equivalent widths is consistent with previous measurements of the column density distribution (Ellison et al. 2000; Songaila 2001; Scannapieco et al. 2006a).

2. We counted pairs of $C_{\text {IV }}$ systems as a function of their separation. The ratio of these counts to the number of pairs in simulated sightlines determined the clustering strength (at each separation). The large clustering amplitude, $\xi>1$, required special treatment of the errors. In an Appendix, we outlined how to compute a correction to the Poisson term that characterizes the Landy \& Szalay (1993) correlation estimator. We learned that reducing the uncertainty at fixed separation will require more systems per sightline, i.e., more sensitive observations rather than more quasars.

3 . We examined the correlation of C IV systems between sightlines. When a $\mathrm{C}$ IV system is present in sightline $A$, we found a better than average chance of finding a C IV system within $1000 \mathrm{~km} \mathrm{~s}^{-1}$ in sightline $B$. The correlation amplitude grows as the separation of the sightlines decreases. We presented a projection of the $\mathrm{C}$ IV correlation function over transverse scales from $0.075 \leqslant r_{p}\left(h^{-1}\right.$ comoving $\left.\mathrm{Mpc}\right) \leqslant 2.84$. The fitted break scale, which defines the radius of an enriched region, was found to be sensitive to the correlation length. Taking the prior $r_{0} \equiv 4.0 h^{-1}$ comoving Mpc, we found $r_{1}=0.42 \pm 0.15 h^{-1}$ comoving Mpc. However, larger enriched regions and correlation lengths improved the fit statistic slightly, e.g., $r_{1}=0.74 \pm 0.21 h^{-1}$ comoving Mpc and $r_{0}=4.9 h^{-1}$ comoving Mpc. Repeating our analysis with more widely separated sightlines will break this degeneracy. 
4. The traditional method for estimating the coherence length of enriched regions breaks down due to galaxy-galaxy clustering. We demonstrated that the result cannot be trusted when the most-likely diameter equals the separation of the widest binary presenting C IV systems at similar redshift. We propose a revised maximum-likelihood analysis. When applied to our data, it converges to a coherence length of $0.3 h^{-1}$ comoving Mpc. This result is consistent with our interpretation of the transverse correlation function.

5. We measured the clustering of C IV systems along single sightlines. We confirmed that the correlation function flattens at small velocity separation as suggested previously (Rauch et al. 1996; Pichon et al. 2003; Scannapieco et al. 2006a). Neglecting peculiar velocities, we fitted a correlation length, $r_{0}=4.9 \pm 0.7 h^{-1}$ comoving Mpc, and break, $r_{1}=1.2 \pm 0.3 h^{-1}$ comoving Mpc (where $\gamma \equiv 1.6$ ). Since the correlation length was consistent with that fitted to galaxies, we adopted $r_{0}=4.0 h^{-1}$ comoving Mpc (Adelberger et al. 2005a) and fit $r_{1}=1.08 \pm 0.17 h^{-1}$ comoving Mpc. The break scale in redshift space is larger than the break required by the transverse C IV correlation function. We attribute the difference to random, peculiar velocities that impact the line-of-sight correlation function but have no affect on the transverse correlation function.

6. We parameterized the line-of-sight velocity distribution for the enriched regions and fitted the line-of-sight correlation function with $r_{0}=4.0 \mathrm{~h}^{-1}$ comoving Mpc $r_{1}=0.42$ $h^{-1}$ comoving Mpc. The resulting rms peculiar velocity was $a=120 \mathrm{~km} \mathrm{~s}^{-1}$ with a $3 \sigma$ upper limit of $300 \mathrm{~km}$ $\mathrm{s}^{-1}$. Larger velocities introduced more power at separations $\Delta v \geqslant a$ than we measure. These speeds may entirely reflect the relative motions of galaxies. Because the scale $r_{1}$ is somewhat larger than the virial radius, virial motion within galaxies seem less likely to dominate. Most importantly, however, we conclude that the typical speeds of outflows over the enriched regions are $\lesssim 200 \mathrm{~km} \mathrm{~s}^{-1}$. The maximum outflow speed is even lower if the correlation length $r_{0}$ is larger than assumed.

7. We measure a higher clustering amplitude for C IV absorbers at a median redshift of 2.5 than we do at redshift 3.3. This growth in clustering amplitude with cosmic time is consistent with the gravitational collapse of large structures. The measurement uncertainties allow ongoing enrichment however.

Common $\mathrm{C}$ IV systems in spectra of binary quasars require enriched regions at least $0.42 \mathrm{~h}^{-1}$ comoving Mpc in radius. This scale for enriched regions is larger than impact parameters at which Steidel et al. (2010) find C IV absorption. We attribute the larger size to our higher sensitivity to weak C IV systems. Small, but significant, redshift-space distortions explain why the lineof-sight correlation function flattens on a larger scale. If outflows contribute significantly to these peculiar velocities, the outflow velocity could be as high as $200 \mathrm{~km} \mathrm{~s}^{-1}$. At $z \simeq 3$, our smallest fitted size and largest fitted velocity give a minimum timescale to disperse the observed metals. This timescale exceeds the median age of a Lyman-break galaxy.

In our qualitative picture, galactic winds disperse metals out to a radius where the outflow stalls or falls back toward the galaxy. If these bubbles of metals do not overlap, then the size of an enriched region measures the turnaround radius of the galactic wind. Large bubbles of metals can be made consistent with lower outflow speeds by placing the enrichment at a considerably earlier epoch. For example, bubbles blown by galactic winds at $z \gtrsim 6$, need only reach a physical radius of $86 \mathrm{kpc}$ to spread metals over $0.42 h^{-1}$ comoving Mpc by redshift 3 . The expansion of the universe aids the dispersal of metals in this picture.

The correlation length of the CIV systems only requires the source of the metals be highly biased like Lyman-break galaxies (Scannapieco 2005; Scannapieco et al. 2006a). Lower mass galaxies at higher redshift are naturally highly clustered (Scannapieco 2005). The lower halo mass of early galaxies facilitates the escape of metals (Dekel \& Silk 1986; Martin 1999). Their large number density may lead to bubble coalescence enlarging the scale of metal-enriched regions well beyond that of an individual bubble.

If the metals detected in these $\mathrm{C}$ IV systems were not dispersed by galaxies at $z \simeq 3$, then how much earlier did dispersal occur? The sizes of the enriched regions measured here provide a new reality check for cosmological simulations that address cosmic chemical evolution (e.g., Wiersma et al. 2009; Oppenheimer \& Davé 2008; Kobayashi et al. 2007; Scannapieco et al. 2006b). Some simple arguments, however, suggest $z \gtrsim 4.3$. Consider galaxies at $z=4.3$ with the same bias as Lyman-break galaxies. The halo mass of such galaxies would be about 0.2 times that of a typical Lyman-break galaxy. The comoving space density of such halos is about five times higher than that of Lyman-break galaxies. In the $723 \mathrm{Myr}$ between redshift 4.3 and 3.0, an outflow traveling at $200 \mathrm{~km} \mathrm{~s}^{-1}$ would reach $0.55 \mathrm{~h}^{-1}$ comoving Mpc. Some of these galaxies would still be around at redshift 3 and recognizable by their older stellar population and low mass. (The expansion rate of the wind exceeds the cosmic expansion rate, so the wind is unlikely to stall.) Taking a cross section of $\pi r_{1}^{2}$, we estimate the density of C IV systems (down to our sensitivity of $20 \mathrm{~m} \AA$ ) would be $d N / d z \sim 5$. This value is comparable to our data if there are 2-3 C IV system per galaxy. We obtain higher $d N / d z$, and therefore more systems per galaxy if we place enrichment at higher redshift. Only of order $10 \%$ of the C IV population is easily attributed to enrichment at redshift 3 by Lyman-break galaxies.

The results described above illustrate how quasar tomography can be used to map the distribution of intergalactic metals. The sightlines used in this paper were chosen for the relatively small transverse separation of the binary quasars. It is possible to observe binaries at wider separation. When the correlation of metal-line systems is measured over a very broader range in scale, it will be possible to simultaneously fit $r_{1}, r_{0}$, and $\gamma$ to the transverse correlation function. The feasibility of making sensitive observations along many pairs of sightlines will grow enormously with the next generation of giant ground-based telescopes. Our results suggest that measuring the evolution in the size of enriched regions with redshift would directly reveal the IGM enrichment history.

We thank Nicolas Bouché, Jon Oiler, and Rob Thacker for discussions that improved the content of this paper. C.L.M. acknowledges support for this work from the David and Lucile Packard Foundation and NSF grant AST-080816. E.S. acknowledges support from NASA theory grant NNX09AD10G. S.G.D. acknowledges partial support from NSF grants AST-0407448 and AST-0909182, and the Ajax Foundation. The authors wish to recognize and acknowledge the very significant cultural role and reverence that the summit of Mauna Kea has always had within the indigenous Hawaiian community. We are most fortunate to have the opportunity to conduct observations from this 
mountain. We thank Mr. Kurt Soto, Dr. Grant Hill, and Dr. Greg Wirth for assistance during the observing runs.

Facilities: Keck:II

\section{APPENDIX}

\section{COMPUTING THE VARIANCE OF OUR CORRELATION MEASUREMENTS}

\section{A.1. Single Sightlines}

First, we consider measurements along single lines of sight. Numbering all the sightlines in the sample with an index $\ell$, we can compute the variance in the correlation function for a bin $k$, representing separations from $v-\Delta v / 2$ to $v+\Delta v / 2$ as

$$
\sigma^{2}\left(\xi_{k}\right)=\frac{1}{\sum_{l}\langle R R\rangle_{k}^{\ell}} \times \sum_{l} \sigma^{2}\left(\langle D D\rangle_{k}^{\ell}\right)
$$

where $\langle D D\rangle_{k}^{\ell}$ is the measured number of pairs in sightline $\ell$ and bin $k,\langle R R\rangle_{k}^{\ell}$ is the expected number of pairs in sightline $\ell$ and bin $k$ for a random distribution, such that $\sum_{\ell}\langle R R\rangle_{k}^{\ell}$ is the total number of expected pairs in bin $k$ in the full data set, and $\sigma^{2}\left(\langle D D\rangle_{k}^{\ell}\right)$ is the variance in $\langle D D\rangle_{k}^{\ell}$.

From Poisson statistics $\sigma^{2}\left(\langle D D\rangle_{k}^{\ell}\right)=\langle D D\rangle_{k}^{\ell}$, however, as discussed by Mo et al. (1992), this is an underestimate of the full variance. To compute the full variance of a single sightline we divide it into many small redshift bins with an index $i$. For simplicity, we drop the superscript $\ell$ and let $N_{i}$ be the number of absorbers in one of these bins, where the bins are small so that $N_{i}$ is always zero or 1 . To compute the number of pairs in a given sightline, one must compute $\langle D D\rangle_{k}=\frac{1}{2} \sum_{i, j} N_{i} N_{j} U_{i, j}$ where $U_{i, j}$ is 1 if the velocity difference between line-of-sight bins $i$ and $j$ is greater than $v-\Delta v / 2$ and less than $v+\Delta v / 2$, and $U_{i, j}=0$ otherwise.

If we square this, we can get an estimate of the variance

$$
\frac{1}{4} \sum_{i, j, m, n} N_{i} N_{j} N_{m} N_{n} U_{i, j} U_{m, n}
$$

Here there are three possibilities, one is that $i, j, m$, and $n$ are all different, one is that either $i$ or $j$ falls in the same bin as either $m$ or $n$, and one is that both $i$ and $j$ are matched with $m$ and $n$. This gives us three terms. If all the bins are different, this gives the average correlation function squared, which does not contribute to the variance. If there are two matches between bins, this gives a term that goes as the number of pairs, which leads to the Poisson term. Finally, if there is only one match, we obtain a term of the form

$$
\sum_{i, j, m}\left\langle N_{i} N_{j} N_{m}\right\rangle U_{i, j} U_{i, m}
$$

There is no factor of $1 / 4$ here because there are four possible pairings that contribute to this term. The term is equal to the joint probability of finding three absorbers: two of them at a distance $v \pm \Delta v / 2$ from the first one.

There are two possibilities for this distribution. One in which absorption-line systems $j$ and $m$ are on opposite sides of absorber $i$. This possibility contributes

$$
N_{\mathrm{abs}} \times\{2 n \Delta v[1+\xi(v)]\} \times\{n \Delta v[1+\xi(v)]\},
$$

where $N_{\text {abs }}$ is the total number of absorption-line systems in a given sightline and $n$ is the average number of absorption-line systems per unit length. There is a 2 in the second term because absorber $j$ can either lie at higher or lower redshift than absorber $i$, but this factor does not appear in the third term because we are assuming that $j$ and $m$ are on opposite sides. Note that we are also ignoring the three-point correlation function, the (usually small) excess probability of any absorber being located in bin $k$ above due to both $i$ and $j$.

The second possibility for Equation (A3) is that absorptionline systems $j$ and $m$ are on the same side of absorber $i$. In this case, it is more important that $j$ and $m$ are near each other than at a distance $v$ from absorber $i$. So, this contribution looks like

$$
N_{\mathrm{abs}} \times\{2 n \Delta v[1+\xi(v)]\} \times\{n \Delta v[1+\overline{\bar{\xi}}(v)]\}
$$

where we define

$$
\overline{\bar{\xi}}(\Delta v)=\left[\Delta v^{-2} \int_{0}^{\Delta v} d v^{\prime} \int_{0}^{\Delta v} d v^{\prime \prime} \xi\left(v^{\prime}-v^{\prime \prime}\right)\right],
$$

where the double over bar indicates an average over two positions, all positions in which absorption-line systems $j$ and $m$ could lie within a bin of size $\Delta v$. Again, we have ignored the contribution from the three-point function, now considering only the excess probability due to the fact that $j$ and $m$ are in the same bin.

Summing up these two contributions and factoring out $\langle D D\rangle_{k}^{\ell}$, where

$$
\langle D D\rangle_{k}=\frac{N_{\mathrm{abs}}}{2}\{2 n \Delta v[1+\xi(v)]\}
$$

gives

$$
\sum_{i, j, m}\left\langle N_{i} N_{j} N_{m}\right\rangle U_{i, j} U_{i, m}=2\langle D D\rangle_{k} 2 n \Delta v\left[1+\frac{\xi(v)+\overline{\bar{\xi}}(\Delta v)}{2}\right]
$$

such that the total variance in sightline $\ell$ is given by

$$
\sigma^{2}\left(\langle D D\rangle_{k}^{\ell}\right)=\langle D D\rangle_{k}^{\ell}+\frac{4\left(\langle D D\rangle_{k}^{\ell}\right)^{2}}{N_{\mathrm{abs}}}\left\{1+\frac{\overline{\bar{\xi}}(\Delta v)-\xi(v)}{2[1+\xi(v)]}\right\} .
$$

In the case in which the correlation function is small, the term in the brackets is just 1 , recovering the extra contribution to the variance as computed in Mo et al. (1992); but if we know $\xi(v)$, then we can also evaluate the term in brackets.

This variance can be reduced by working with the Landy and Szalay estimator for the correlation function, which for a single sightline is given by $\frac{D D-2 D R+R R}{R R}$. To compute the variance of this estimator, we have to consider two additional terms. The first of these arises from the cross-correlation between $D D$ and $D R$ takes the form

$$
-2 \sum_{i, j, k, m} N_{i} N_{j} N_{m} R_{n} U_{i, j} U_{m, n},
$$

where there is a 2 because only the $\langle D D\rangle$ term is defined with a $1 / 2$ in front. Again we have a term in which $i, j, m$, and $n$ are all different which does not contribute to the variance, and a term in which $i$ and $m$ or $j$ and $m$ are paired, which gives

$$
-4 \sum_{i, j, n}\left\langle N_{i} N_{j} R_{n}\right\rangle U_{i, j} U_{i, n}
$$


When appropriately normalized to account for the number of absorption-line systems in the random data set

$$
-2 N_{\mathrm{abs}}\left[2 n \Delta v(1+\xi(v)] 2 n \Delta v=-4\langle D D\rangle_{k} 2 n \Delta v .\right.
$$

Finally, from the term $4\langle D R D R\rangle$ we have a term in which $N_{i}$ and $N_{k}$ are pared which, when normalized, gives

$$
N_{\mathrm{abs}}[2 n \Delta v]^{2}=2\langle D D\rangle_{k} \frac{2 n \Delta v}{1+\xi(v)} .
$$

Putting this together, we get that for the Landy and Szalay estimator, for a single sightline, the total variance is equal to the Poisson term, plus a second a term

$$
2\langle D D\rangle_{k} 2 n \Delta v\left[-1+\frac{1}{1+\xi(v)}+\frac{\xi(v)+\overline{\bar{\xi}}(\Delta v)}{2}\right],
$$

such that the variance in sightline $\ell$ is given by

$$
\begin{aligned}
\sigma^{2}\left(\langle D D\rangle_{k}^{\ell}\right)= & \langle D D\rangle_{k}^{\ell}+\frac{4\left(\langle D D\rangle_{k}^{\ell}\right)^{2}}{N_{\mathrm{abs}}} \\
& \times\left[\left(\frac{\xi(v)}{1+\xi(v)}\right)^{2}+\frac{\overline{\bar{\xi}}(\Delta v)-\xi(v)}{2(1+\xi(v))}\right] .
\end{aligned}
$$

We do not know the correlation function to evaluate Equations (A9) and (A15), but as an upper limit on the variance we can take the galaxy-galaxy correlation function, taking into account the resolution of our data. This is a power law with a slope $\gamma$ and correlation length $v_{0} \equiv H(z) r_{0}$, above $v_{\text {res }}=50$ $\mathrm{km} \mathrm{s}^{-1}$, and 0 below that. That is

$$
\xi(v)=\left(v / v_{0}\right)^{-\gamma},
$$

if $v \geqslant v_{\text {res }}$ and $\xi(v)=0$ otherwise, where $r_{0}$ and $\gamma$ are taken to fit the galaxy-galaxy correlation function. In this case,

$$
\begin{aligned}
\overline{\bar{\xi}}(\Delta v)= & \left(\frac{\Delta v}{v_{0}}\right)^{-\gamma} \frac{2}{(2-\gamma)(1-\gamma)}+\left(\frac{v_{\text {res }}}{v_{0}}\right)^{-\gamma} \\
& \times\left[\left(\frac{v_{\text {res }}}{\Delta v}\right)^{2} \frac{2}{(2-\gamma)}-\frac{v_{\text {res }}}{\Delta v} \frac{2}{1-\gamma}\right],
\end{aligned}
$$

if $\Delta v \geqslant v_{\text {res }}$ and $\overline{\bar{\xi}} \Delta v=0$ otherwise.

\section{A.2. Pairs of Sightlines}

Moving on to the transverse correlation function between two sightlines $A$ and $B$ that make up a pair $\ell$, the simplest estimator is

$$
1+\xi_{k}^{T}=\frac{1}{\sum_{\ell \in k}\left\langle R^{A} R^{B}\right\rangle} \sum_{\ell \in k}\left\langle D^{A} D^{B}\right\rangle^{\ell},
$$

where the sum is over all pairs $\ell$ whose separations put them in a traverse bin $k$. The case we are interested in is the one in which $\left\langle D^{A} D^{B}\right\rangle^{\ell}$ includes all absorption-line systems that are separated by $0 \mathrm{~km} \mathrm{~s}^{-1}$ up to some maximum value, $\Delta v$.

Let us again consider the variance for a single sightline. Following our notation from the previous section, we define $N_{\mathrm{abs}}^{A}$ and $N_{\text {abs }}^{B}$ as the number of absorption-line systems in sightline
$A$ and $B$ in this pair, and $n^{A}$ and $n^{B}$ as the number density of absorption-line systems in sightline $A$ and $B$. In this case

$$
\begin{aligned}
\left\langle D^{A} D^{B}\right\rangle^{\ell} & =N_{\text {abs }}^{A} 2 \Delta v n^{B}\left[1+\bar{\xi}\left(r_{p}, \Delta\right)\right] \\
& =N_{\text {abs }}^{B} 2 \Delta v n^{A}\left[1+\bar{\xi}\left(r_{p}, \Delta v\right)\right],
\end{aligned}
$$

where $\bar{\xi}\left(r_{p}, \Delta v\right) \equiv \frac{1}{\Delta v} \int_{0}^{\Delta v} d v^{\prime} \xi\left(r_{p}, v^{\prime}\right)$, such that the single bar denotes a single velocity average of $\xi\left(r_{p}, v^{\prime}\right)$ the correlation function between two absorption-line systems at a transverse comoving distance of $r_{p}$ and comoving separation of $v^{\prime}$.

To compute the variance of a single pair of sightlines, we again divide both sightlines into many small bins and square this gives us

$$
\sum_{i, j, m, n} N_{i}^{A} N_{j}^{B} N_{m}^{A} N_{n}^{B} U_{i, j} U_{m, n}
$$

This leads to a term where $i, j, m$ and $n$ are all different, which gives the mean value squared, a term where $i=m$ and $j=n$, which corresponds to the Poisson term, and a new term

$$
\sum_{i, m, n}\left\langle N_{i}^{A} N_{m}^{A} N_{n}^{B}\right\rangle U_{i, n} U_{m, n}+\sum_{i, j, n}\left\langle N_{i}^{A} N_{j}^{B} N_{n}^{B}\right\rangle U_{i, m} U_{j, m},
$$

Focusing on the first of the two sums in Equation (A21), we can simplify this by considering only the case in which $i$ is at a lower redshift than $n$, and then multiplying by 2 . This gives

$$
\begin{aligned}
& \sum_{i, n, m}\left\langle N_{i}^{A} N_{m}^{A} N_{n}^{B}\right\rangle U_{i, m} U_{n, m} \\
& =N_{\mathrm{abs}}^{B}\left\{2 n^{A} \Delta v\left[1+\bar{\xi}\left(r_{p}, \Delta v\right)\right]\right\}\left\{2 n^{A} \Delta v\left[1+\overline{\bar{\xi}}\left(r_{p}, \Delta v\right)\right]\right\},
\end{aligned}
$$

where

$$
\begin{aligned}
\overline{\bar{\xi}}\left(r_{p}, \Delta v\right) \equiv & \frac{1}{\left[1+\bar{\xi}\left(r_{p}, \Delta v\right)\right] 2(\Delta v)^{2}} \int_{0}^{\Delta v} d v^{\prime}\left[1+\xi\left(r_{p}, v^{\prime}\right)\right] \\
& \times \int_{-\Delta v}^{\Delta v} d v^{\prime \prime}\left\{\max \left[\xi\left(r_{p}, v^{\prime \prime}\right), \xi\left(0,\left|v^{\prime \prime}-v^{\prime}\right|\right)\right]\right\} .
\end{aligned}
$$

Factoring out $\left\langle D^{A} D^{B}\right\rangle^{\ell}$ this gives

$$
\sum_{i, n, m}\left\langle N_{i}^{A} N_{n}^{A} N_{m}^{B}\right\rangle U_{i, m} U_{n, m}=\left\langle D^{A} D^{B}\right\rangle^{\ell} 2 n^{A} \Delta v\left[1+\overline{\bar{\xi}}\left(r_{p}, \Delta v\right)\right] .
$$

Computing the second term in Equation (A21) in a similar manner we get

$$
\sum_{i, j, m}\left\langle N_{i}^{A} N_{j}^{B} N_{m}^{B}\right\rangle U_{i, j} U_{i, m}=\left\langle D^{A} D^{B}\right\rangle^{\ell} 2 n^{B} \Delta v\left[1+\overline{\bar{\xi}}\left(r_{p}, \Delta v\right)\right] .
$$

Thus, the variance for pair $\ell$ is

$$
\begin{aligned}
\sigma^{2}\left(\left\langle D^{A} D^{B}\right\rangle^{\ell}\right)= & \left\langle D^{A} D^{B}\right\rangle^{\ell}+\left(\left\langle D^{A} D^{B}\right\rangle^{\ell}\right)^{2}\left[\frac{1}{N_{A}}+\frac{1}{N_{B}}\right] \\
& \times\left[1+\frac{\overline{\bar{\xi}}\left(r_{p}, \Delta v\right)-\bar{\xi}\left(r_{p}, \Delta v\right)}{1+\bar{\xi}\left(r_{p}, \Delta v\right)}\right] .
\end{aligned}
$$


In the case of the Landy and Szalay estimator, which now goes as $\frac{D^{A} D^{B}-D^{A} R^{B}-R^{A} D^{B}+R^{A} R^{B}}{R^{A} R^{B}}$, two new cross terms arise. The first is a term of the form $-2\left(\left\langle D^{A} D^{B} D^{A} R^{B}\right\rangle+\left\langle D^{A} D^{B} R^{A} D^{B}\right\rangle\right)$ which leads to

$$
\begin{aligned}
& -2\left(\sum_{i, j, m} N_{i}^{A} N_{j}^{B} R_{m}^{A} U_{i, j} U_{j, m}+\sum_{i, j, n} N_{i}^{A} R_{j}^{B} N_{n}^{B} U_{i, j} U_{j, n}\right) \\
& =-2\left\langle D^{A} D^{B}\right\rangle^{\ell} 2 \Delta v\left(n^{A}+n^{B}\right) .
\end{aligned}
$$

The second is a term $\left\langle D^{A} R^{B} D^{A} R^{B}\right\rangle+\left\langle R^{A} D^{B} R^{A} D^{B}\right\rangle$, which leads to

$$
\begin{aligned}
& \left(\sum_{i, j, m} R_{i}^{A} R_{m}^{A} N_{j}^{B} U_{i, j} U_{j, m}+\sum_{i, j, m, n} N_{i}^{A} R_{m}^{B} N_{j}^{B} U_{i, j} U_{j, m}\right) \\
& =\left\langle D^{A} D^{B}\right\rangle^{\ell} 2 \Delta v \frac{\left(n^{A}+n^{B}\right)}{1+\bar{\xi}\left(r_{p}, \Delta v\right)} .
\end{aligned}
$$

Summing these together then gives

$$
\begin{gathered}
\sigma^{2}\left(\left\langle D^{A} D^{B}\right\rangle^{\ell}\right)=\left\langle D^{A} D^{B}\right\rangle^{\ell}+\left(\left\langle D^{A} D^{B}\right\rangle^{\ell}\right)^{2}\left[\frac{1}{N_{A}}+\frac{1}{N_{B}}\right] \\
\quad \times\left[\left(\frac{\bar{\xi}\left(r_{p}, \Delta v\right)}{1+\bar{\xi}\left(r_{p}, \Delta v\right)}\right)^{2}+\frac{\overline{\bar{\xi}}\left(r_{p}, \Delta v\right)-\bar{\xi}\left(r_{p}, \Delta v\right)}{1+\bar{\xi}\left(r_{p}, \Delta v\right)}\right] .(\mathrm{A}
\end{gathered}
$$

In the case of our data, we can estimate $\bar{\xi}\left(r_{p}, \Delta v\right)$ and $\overline{\bar{\xi}}\left(r_{p}, \Delta v\right)$ by carrying out appropriate integrals over

$$
\xi\left(r_{p}, v\right)=\left[\frac{r_{p}^{2}+\Pi^{2}}{r_{0}^{2}}\right]^{-\gamma / 2}
$$

if $v \geqslant v_{\text {res }}$ or $r_{p} \neq 0$ and $\xi\left(r_{p}, v\right)=0$ otherwise, where $\Pi=v / H(z)$ and $r_{0}$ and $\gamma$ are taken to fit the galaxy-galaxy correlation function.

\section{REFERENCES}

Adelberger, K. L., et al. 2005a, ApJ, 619, 697

Adelberger, K. L., et al. 2005b, ApJ, 629, 636

Aguirre, A., et al. 2001, ApJ, 560, 599

Becker, G., Rauch, M., \& Sargent, W. L. W. 2009, ApJ, 698, 1010

Cooksey, K. L., Thom, C., Prochaska, J. X., \& Chen, H.-W. 2010, ApJ, 708, 868

Cooray, A., \& Sheth, R. 2002, Phys. Rep., 372, 1

Coppolani, F., et al. 2006, MNRAS, 370, 1804

Crotts, A. P. S., et al. 1994, ApJ, 437, L79

Davis, M., \& Peebles, J. E. 1983, ApJ, 267, 465

Dekel, A., \& Birnboim, Y. 2008, MNRAS, 383, 119
Dekel, A., \& Silk, J. 1986, ApJ, 303, 39

Dinshaw, N., Weymann, R. J., Impey, C. D., Foltz, C. B., Morris, S. L., \& Ake, T. 1997, ApJ, 491, 45

D'Odorico, V., et al. 2010, MNRAS, 401, 2715

Ellison, S. L., et al. 2004, A\&A, 414, 79

Ellison, S., et al. 2000, AJ, 120, 1175

Ferrara, A., Pettini, M., \& Shchekinov, Y. 2000, MNRAS, 319, 539

Furlanetto, S. R., \& Loeb, A. 2003, ApJ, 588, 18

Gnedin, \& Ostriker 1997, ApJ, 486, 581

Hawkins, E., et al. 2003, MNRAS, 346, 78

Hennawi, J. F. 2004, PhD thesis, Princeton Univ.

Hennawi, J. F., et al. 2006a, AJ, 131, 1

Hennawi, J. F., et al. 2006b, ApJ, 651, 61

Hennawi, J. F., et al. 2010, ApJ, 719, 1672

Hildebrandt, H., et al. 2009, A\&A, 498, 725

Kawata, D., \& Rauch, M. 2007, ApJ, 663, 38

Kobayashi, C., et al. 2007, MNRAS, 376, 1465

Kriss, G. 1994, in ASP Conf. Ser. 61, Astronomical Data Analysis Software \& Systems III, ed. D. R. Crabtree, R. J. Hanisch, \& J. Barnes (San Francisco, CA: ASP), 437

Landy, S. D., \& Szalay, A. S. 1993, ApJ, 412, 64

Lopez, S., et al. 2000, A\&A, 357, 37

Lu, L. 1991, ApJ, 379, 99

Madau, P., Ferrara, A., \& Rees, M. J. 2001, ApJ, 555, 92

Martin, C. L. 1999, ApJ, 513, 156

Martin, C. L. 2005, ApJ, 621, 227

Martin, C. L., \& Bouché, N. 2009, ApJ, 703, 1394

McGill, C. 1990, MNRAS, 242, 544

Meyer, D. M., \& York, D. G. 1987, ApJ, 315, 5

Mo, H. J., Jing, Y. P., \& Börner, G. 1992, ApJ, 392, 452

Morton, D. 2003, ApJS, 149, 205

Oppenheimer, B. D., \& Davé, R. 2008, MNRAS, 387, 577

Oppenheimer, B. D., Davé, R., \& Finlator, K. 2009, MNRAS, 396, 729

Petitjean, P., \& Bergeron, J. 1990, A\&A, 231, 309

Pettini, M., et al. 2002, ApJ, 569, 742

Pichon, C., et al. 2003, ApJ, 597, L97

Porciani, C., \& Madau, P. 2005, ApJ, 625, 43

Qian, Y.-Z., Sargent, W. L. W., \& Wasserburg, G. J. 2002, ApJ, 569, 61

Rauch, M., Sargent, W. L. W., \& Barlow, T. A. 1999, ApJ, 515, 500

Rauch, M., Sargent, W. L. W., \& Barlow, T. A. 2001, ApJ, 554, 823

Rauch, M., Sargent, W. L. W., Womble, D. S., \& Barlow, T. A. 1996, ApJ, 467,

Rauch, M., et al. 2002, ApJ, 576, 45

Rupke, D., et al. 2005, ApJS, 160, 115

Ryan-Weber, E. V., Pettini, M., Madau, P., \& Zych, B. J. 2009, MNRAS, 395, 1476

Sawicki, M., \& Yee, H. C. 1998, AJ, 115, 1329

Scannapieco, E. 2005, ApJ, 624, 1

Scannapieco, E., Ferrara, A., \& Madau, P. 2002, ApJ, 574, 590

Scannapieco, E., et al. 2006a, MNRAS, 365, 615

Scannapieco, E., et al. 2006b, MNRAS, 371, 1125

Schaye, Y., et al. 2003, ApJ, 596, 768

Shapley, A. E., et al. 2001, ApJ, 562, 95

Shapley, A. E., et al. 2003, ApJ, 588, 65

Sheinis, A. I., et al. 2002, PASP, 114, 851

Shen, Y., et al. 2007, AJ, 133, 2222

Songaila, A. 2001, ApJ, 561, L154

Songaila, A., \& Cowie, L. L. 1996, AJ, 112, 335

Steidel, C. C., et al. 2010, ApJ, 717, 289

Theuns, T., et al. 2002, ApJ, 578, L5

Tytler, D., et al. 2009, MNRAS, 392, 1539

Weiner, B. J., et al. 2009, ApJ, 692, 187

Wiersma, R. P. C., et al. 2009, MNRAS, 399, 574 